\title{
DIPG in children - what can we learn from the past?
}

\author{
Magimairajan Issai Vanan ${ }^{1,2 *}$ and David D. Eisenstat ${ }^{3,4,5 *}$ \\ 'Department of Pediatrics and Child Health, University of Manitoba, Winnipeg, MB, Canada, ${ }^{2}$ Department of Biochemistry \\ and Medical Genetics, University of Manitoba, Winnipeg, MB, Canada, ${ }^{3}$ Department of Pediatrics, University of Alberta, \\ Edmonton, AB, Canada, ${ }^{4}$ Department of Medical Genetics, University of Alberta, Edmonton, AB, Canada, ${ }^{5}$ Department of \\ Oncology, University of Alberta, Edmonton, AB, Canada
}

\section{OPEN ACCESS}

Edited by:

André O. Von Bueren,

University Medical Center Göttingen,

Germany

Reviewed by:

Katherine Warren,

National Cancer Institute, USA Jacques Grill,

Gustave Roussy Cancer Institute,

France

Ofelia Cruz,

Hospital Sant Joan de Déu, Spain

*Correspondence:

Magimairajan Issai Vanan mivanan@cancercare.mb.ca;

David D. Eisenstat eisensta@ualberta.ca

Specialty section: This article was submitted to Neuro-Oncology, a section of the journal Frontiers in Oncology

Received: 07 June 2015 Accepted: 08 October 2015 Published: 21 October 2015

Citation:

Vanan MI and Eisenstat DD (2015) DIPG in children - what can we learn from the past?

Front. Oncol. 5:237.

doi: 10.3389/fonc.2015.00237
Brainstem tumors represent $10-15 \%$ of pediatric central nervous system tumors and diffuse intrinsic pontine glioma (DIPG) is the most common brainstem tumor of childhood. DIPG is almost uniformly fatal and is the leading cause of brain tumor-related death in children. To date, radiation therapy $(R T)$ is the only form of treatment that offers a transient benefit in DIPG. Chemotherapeutic strategies including multi-agent neoadjuvant chemotherapy, concurrent chemotherapy with RT, and adjuvant chemotherapy have not provided any survival advantage. To overcome the restrictive ability of the intact blood-brain barrier (BBB) in DIPG, several alternative drug delivery strategies have been proposed but have met with minimal success. Targeted therapies either alone or in combination with RT have also not improved survival. Five decades of unsuccessful therapies coupled with recent advances in the genetics and biology of DIPG have taught us several important lessons (1). DIPG is a heterogeneous group of tumors that are biologically distinct from other pediatric and adult high grade gliomas (HGG). Adapting chemotherapy and targeted therapies that are used in pediatric or adult HGG for the treatment of DIPG should be abandoned (2). Biopsy of DIPG is relatively safe and informative and should be considered in the context of multicenter clinical trials (3). DIPG probably represents a whole brain disease so regular neuraxis imaging is important at diagnosis and during therapy (4). BBB permeability is of major concern in DIPG and overcoming this barrier may ensure that drugs reach the tumor (5). Recent development of DIPG tumor models should help us accurately identify and validate therapeutic targets and small molecule inhibitors in the treatment of this deadly tumor.

Keywords: diffuse intrinsic pontine glioma, blood-brain barrier, pediatric neuro-oncology, brain tumor, reirradiation

\section{INTRODUCTION}

The anatomic brainstem includes three distinct regions: the midbrain, pons, and medulla oblongata. Tumors of the brainstem constitute approximately $10-15 \%$ of all central nervous system (CNS) tumors in children ages $0-14$ years in the USA; the majority of these tumors are of glial origin (gliomas) (1). Prior to modern imaging techniques, all brainstem gliomas were regarded as a single pathological entity, and the prognosis was considered uniformly poor. Computerized tomography (CT) and Magnetic Resonance Imaging (MRI) have been used to classify brainstem tumors based on their growth pattern and amenability to surgical resection. Diffuse intrinsic pontine gliomas (DIPG) 
are the most common brainstem tumors in children and account for greater than $80 \%$ of brainstem gliomas in this age group. The widespread infiltrative nature of this tumor, predominantly highgrade features, coupled with its critical anatomic location and inoperability, has led to a uniformly poor outcome in children with DIPG.

\section{Clinical Features/Diagnosis}

DIPG occurs in all age groups but is most commonly diagnosed in children between the ages of 5 and 10 years. There is equal predilection for both sexes $(\mathrm{M}: \mathrm{F}=1: 1)$, and the prognosis is significantly worse than that of other brainstem tumors. Due to local infiltration and brainstem localization, DIPGs are not amenable to surgical resection. The clinical features of patients with brainstem tumors vary with respect to tumor location and the nature/pattern of growth of these tumors. Patients with DIPG have a short latency (usually $<2-3$ months) between the onset of clinical symptoms and diagnosis. The classic triad of symptoms includes cerebellar signs (e.g., ataxia, dysmetria, dysarthria), longtract signs (e.g., increased tone, hyperreflexia, clonus, Babinski sign, motor deficit, etc.), and isolated or multiple cranial nerve palsies (unilateral or bilateral), more commonly sixth and seventh cranial nerve palsies. Patients with diffuse brainstem tumors associated with neurofibromatosis type 1 (NF1) may mimic DIPG on imaging. However, in the context of NF1, these are usually low-grade gliomas (LGG, WHO grades I-II) that can be asymptomatic or diagnosed in the context of an insidious history of isolated cranial nerve palsy or motor deficit. Careful clinical examination for the stigmata of NF-1 and family history should help in the identification of these lesions that usually do not require any active treatment. In DIPG, signs and symptoms of increased intracranial pressure (due to obstructive hydrocephalus from expansion of the pons) are seen in $<10 \%$ of children. Various other non-specific symptoms present either at the time of diagnosis or at the time of progression include sensory abnormalities, behavioral changes, urinary problems, declining school performance, and respiratory symptoms including sleep apneas.

Over the years, many classification schemes have been proposed for brainstem tumors, and most have utilized the best neuroimaging modalities available at the time of classification (2). The earliest classifications relied on CT and surgical observations; however, the more recent schemes include MRI. In the broadest sense, these tumors are divided into two groups, either focal or diffuse. The more complex schemes subdivide these tumors by location within the brainstem (midbrain, pons, or medulla), growth pattern (intrinsic or exophytic), direction and extent of tumor growth, the presence or absence of contrast enhancement, and the presence of hemorrhage or hydrocephalus (3). In one of the most recent classifications proposed by Choux et al. based on both CT and MRI characteristics, brainstem tumors are divided into four types (4). DIPGs are classified as type I tumors (not to be confused with WHO grade I). These lesions appear hypointense on CT with non-delineated borders and do not significantly enhance on T1-weighted MRI sequences with gadolinium as the contrast reagent. DIPGs are characterized by diffuse infiltration and swelling of the brainstem.

\section{Why will Biopsy of DIPG Become Important?}

Historically, in the pre-CT and MRI eras, stereotactic brainstem biopsies were performed on a routine basis for histological diagnosis of DIPG. Due to the heterogeneity of these tumors, the significant morbidity potentially associated with the biopsies, the limited therapeutic options available based on biopsy results, the prevalence of poor candidates for biopsy at the time of presentation (i.e., those with focal neurological deficits, increased ICP), and the widespread availability of MRI with characteristic imaging findings, routine biopsy as the standard of care was discontinued in the early 1990s (5).

\section{Safety and Feasibility of the Procedure}

Many centers in Europe have been performing routine diagnostic biopsies of children with suspected DIPG. In two of the largest series of brainstem tumor biopsies in children $(6,7)$, there were only transient reversible morbidities (in $<10 \%$ of patients) and no mortality reported. In a retrospective analysis, Cartmill et al. (6) reviewed 18 brainstem glioma patients who underwent CT-guided stereotactic biopsy. All 18 patients had a histological diagnosis of glioma [HGG, $n=13$ (glioblastoma multiforme, $\mathrm{GBM}=8$; anaplastic astrocytoma (AA), $n=5$ ) and low grade gliomas, $n=5$ ]. Besides the five patients who developed a transient increase in their neurological deficits post-operatively (hemiparesis, increased eye movements, and VII nerve palsy), there was no mortality reported. Roujeau et al. (7) reported a prospective series of 24 patients with DIPG who underwent stereotactic biopsy. In two patients, a diagnosis of low-grade gliomas was made (LGG and pilocytic astrocytoma) and each was followed with an initial period of observation before initiating radiotherapy. Only two patients had transient post-operative neurological deficits (cranial nerve palsy and exacerbation of pre-operative hemiparesis) and no deaths were reported. A historical cohort study by Cage et al. (8) of nine patients with DIPG who underwent stereotactic biopsy revealed no intraoperative complications and only one patient with post-operative neurological deficit (seizures with hydrocephalus), not directly related to the procedure. In their review of literature, Cage et al. (8) report $>300$ pediatric cases of DIPG who underwent biopsy with mortality attributable to the procedure in only two cases. In a recent retrospective series from Wang et al., only 3 of the 15 cases of DIPG who underwent biopsy had transient new or worsening neurological deficits and there was no biopsy related mortality (9). Careful pre-operative planning using functional imaging like Diffusion Tensor Imaging tractography to delineate the white matter (especially corticospinal tracts) and PET imaging to identify regions of interest will further decrease the morbidity and increase the diagnostic yield of the biopsy $(10,11)$. The post-operative morbidity of image guided stereotactic biopsy of intrinsic pontine lesions is comparable to biopsy procedures in other brain locations.

\section{Sampling Bias, Diagnostic Yield and Differential Diagnosis}

In all of the biopsy series of DIPG cases (6-9), the pathologic diagnosis revealed low grade as well as HGG. Initial concerns 
regarding the heterogeneity of the tumor leading to sampling bias and downgrading of the tumor (from WHO grade IV to grade II) resulting in less intense therapy, may no longer be considered valid. Recent data from a large series of patients suggests that there are distinct histologic subgroups of DIPG with varying clinical and molecular features (12). Although the patients may present with typical clinical features of DIPG, the histopathology is diverse (spectrum from grade II to IV) and low grade tumors can behave clinically as aggressively as HGGs $(8,12)$. There is potential intra-tumor histologic heterogeneity seen in DIPG as demonstrated in the series by Buczkowicz et al. (12). Tumors with classic GBM histology show grade II or grade III histology in some areas, which may be selected by biopsy. Furthermore, these heterogeneous tumors had classic GBM histologic features in the pons but demonstrated low grade astrocytoma (II) or AA (III) features in the thalamus or brainstem. These observations suggest that histologic grading using current WHO criteria may not be appropriate for diagnostic purposes in DIPG. The diagnostic yield of biopsy specimens in DIPG is very high (90-100\%) (13). Although the amount of tumor tissue obtained is limited by the needle size, it is usually sufficient to make a histopathologic diagnosis. Moreover, recently described biopsy techniques permit multiple biopsies under one anesthetic, thereby providing sufficient tissue not only for diagnosis but also for molecular studies and primary cell culture $(8,13)$. Apart from gliomas (grade II-IV), Primitive Neuro-ectodermal Tumors (PNET) (9, 10,12 ) and rarely germ cell tumors (including germinomas and teratomas) can present as diffuse infiltrative brainstem tumors; the management of these tumor entities is different than for gliomas. Non-malignant conditions presenting as brainstem lesions include neurodegenerative conditions (Alexander disease, acute demyelinating encephalomyelitis, central pontine myelinolysis) and infections (such as paracoccidioidomycosis) (14).

\section{Areas of Controversy}

Some neurosurgeons and other clinicians have introduced the terms typical and atypical DIPG and advocate for biopsy for atypical disease presentations. Literature review on pediatric brainstem gliomas suggests the following criteria for a typical DIPG: a combination of clinical symptoms and signs with short latency <3-6 months - triad of: (1) cerebellar signs, e.g., ataxia, dysmetria and dysarthria; (2) long tract signs: hypertonia, hyperreflexia, motor deficits; and (3) cranial nerve palsies-isolated or multiple sixth, seventh) and radiological findings on MRI: (1) an intrinsic, centrally located tumor involving greater than 50-66\% of the axial diameter of the pons with (2) hypointensity on T1 images, (3) hyperintensity on T2 images with (4) indistinct tumor margins with engulfment of basilar artery and (5) no cystic or exophytic components (15-17). However, a survey conducted among neurosurgeons revealed that there was no consensus regarding what constitutes a typical DIPG on MRI (18). The differential diagnosis for a brain stem tumor in a child with unusual presentation and atypical findings on neuroimaging include embryonal tumors like ATRT, PNET, and non-malignant lesions like infections, neurodegenerative conditions and hemangioblastomas $(9,10,12,14)$; the management of these conditions is entirely different than for gliomas. Focal brainstem tumors usually have insidious onset, prolonged latency and atypical clinical features like failure to thrive, emesis etc. Hence, it may be reasonable for biopsy to be considered in those patients with atypical features for whom clinical management decisions would change when provided with a diagnosis other than DIPG.

In a child with typical imaging appearance of DIPG, the current recommendations are to offer upfront biopsy only in a clinical trial setting (19).

The ability to identify the whole mutational landscape of DIPG using tissue obtained by small-needle pretreatment biopsies (20) coupled with availability of antibodies to detect (a) K27M mutations in both Histone 3 (H3) variants (H3.3 and H3.1) $(21,22)$ and (b) loss of tri-methylation (anti-HEK27me3) due to these mutations provides opportunities to improve diagnosis, assignment of prognosis, and identification of potentially druggable targets (PDGFRA, ACVR1, and FGFR1) (12). There is sufficient evidence in the literature to suggest that as the field moves forward, testing for H3K27M mutations will help in diagnosis $(12,23)$, stratification into subgroups $(12,24)$ and prognosis $(12,23)$ and will be included in the design of prospective clinical trials testing new agents and treatment approaches. In the future, clinical and molecular subgroup-based patient stratification in multi-center clinical trials of DIPG will include stereotactic biopsy when it is considered safe to proceed in individual patients.

\section{IS DIPG A WHOLE BRAIN DISEASE?}

The concept of focal radiotherapy of DIPG is based in part by reports suggesting that the tumor mostly spreads by contiguity and the recurrence is mostly local, within the radiotherapy fields (25-27). Since distant failure is not considered a common phenomenon in the natural history of DIPG, patients do not routinely undergo neuraxis imaging either at diagnosis or follow up after initial therapy. Leptomeningeal dissemination/disease (LMD) was reported in $4-33 \%$ of patients in the pre-MRI era (25-27). In contrast, several studies have identified widespread CNS disease by autopsy and/or neuroimaging (12, 28-35). In fact, disseminated disease may be seen (1) at the time of diagnosis; (2) during follow-up while on treatment; or (3) at the time of autopsy. Benesch et al. (30) in their retrospective data analysis of HGGs report an incidence of 3.1\% ( $N=10 / 546, \mathrm{HGG}=348$, $\mathrm{DIPG}=198$ ) of patients with primary dissemination at diagnosis. However, there was only one case of DIPG $(n=1 / 198,0.5 \%)$ with distant spread (lateral ventricle). Sethi et al. (33) reported $3 / 9$ patients $(33 \%)$ who had LMD detected at diagnosis. In the same series, prospective neuraxis surveillance (MRI of brain and spine) at 4-month intervals after completion of therapy revealed local failure in $75 \%$ of patients (12/15) and LMD in six patients (6/9, 66\%). Moreover, patients with LMD had shorter overall survival (OS) when compared to patients with localized disease: $12.0 \pm 3.3$ months without LMD and $8.0 \pm 2.1$ months with LMD $(P=0.059)$. Donahue et al. (28) reported 15/18 (83\%) children with DIPG with progression of disease of which 14 had local failures. The protocol on which these children were treated mandated monitoring with neuraxis surveillance MRIs every 4 months after completion of treatment. Interestingly, none of these patients had clinical evidence of LMD. The authors concluded that LMD 
occurs primarily in the setting of local progression and therapy should be directed primarily toward improving local control.

Wagner et al. (31) reported secondary disseminating disease (SDD) in DIPG in $13 \%$ of their patients (14/110). The median time between diagnosis and SDD was 7.2 months (range, 4.6 months to 2.2 years) and there was parenchymal metastasis involving the supratentorial, infratentorial, and spinal regions along with LMD. DIPG patients who had LMD and ventricular tumor spread had decreased OS when compared to patients who had parenchymal metastasis. However, SDD did not reduce OS in DIPG tumors, suggesting that rapid local progression of disease is probably the main cause of shortened survival. Gururangan et al. (32) in a retrospective review of neuraxis dissemination in DIPG found metastatic disease in $17.3 \%(18 / 96)$ patients. All the patients died of disease at a median of 5 months (range, 0.5-20) from the diagnosis of neuraxis spread. The pattern of spread showed both LMD and parenchymal involvement. Yoshimura et al. (29) in their autopsy series of DIPG reported more than $50 \%$ of patients had disseminated disease with supratentorial spread in 45\% (18/40) and LMD in 32.5\% (13/40). Almost all the cases except one were HGGs $(\mathrm{GBM}=34, \mathrm{AA}=5)$. Caretti et al. (35) in their autopsy series of 16 DIPG patients found widespread extension involving the midbrain/medulla (63\%), cerebellum (56\%), thalamus (56\%), frontal cortex (25\%), and supratentorial leptomeninges (25\%). There was disease spread into the spinal cord in two out of the three patients examined. A unique form of sub-ventricular spread resulting in tumor nodules seen in the frontal horns of the lateral ventricles was observed in 10 of 16 cases (62.5\%). In one of the largest series of DIPG (12), 38\% of patients exhibited LMD of their tumor. There was parenchymal spread in the form of diffuse involvement of brainstem, spinal cord and supratentorial (thalamus and frontal lobe) regions. There was no association between histology and LMD. However, there were TP53 mutations in 6 of the 11 patients tested and none of the patients with LMD were found to have the ALT (alternate lengthening of telomeres) phenotype. Histone $\mathrm{H} 3$ $\mathrm{K} 27 \mathrm{M}$ mutations in $\mathrm{H} 3.3$ were seen in $67 \%(35 / 52)$ and in $\mathrm{H} 3.1$ were seen in $15 \%(8 / 52)$. Histone $\mathrm{H} 3$ mutations were observed in all histology grades of DIPG (II-IV): $88 \%$ in GBM, $60 \%$ of AA, and $71 \%$ of low grade astrocytomas. K27M-H3.3 mutations were higher in GBM patients (78\%) when compared to AA histology (33\%) $(P=0.0016)$. Importantly, low grade tumors carrying H3.3 mutations had OS similar to that of GBM tumors and patients with high grade tumors with wild-type $\mathrm{H} 3.3$ had OS comparable to wild-type low grade astrocytomas (12).

\section{Diagnostic and Therapeutic Implications}

At the time of diagnosis, whenever available, biopsy specimens of DIPG are suggested to be tested for Histone K27M mutations (H3.3 and 3.1) along with routine WHO histological grading and other markers (TP53, PDGFRA, ACVR1, and FGFR1). This will help in obtaining the correct diagnosis, proper patient selection for clinical trials (for risk stratification and targeted therapies) and prognosis $(12,36)$. Neuroimaging at the time of diagnosis should include MRI of the brain; the spine may be included at the discretion of the attending physician. Thereafter, regular interval neuraxis imaging during follow-up could be included in prospective clinical trials to better understand the natural history of DIPG progression, including disease dissemination. This data may help guide therapy decisions and prognosis.

The majority of DIPG patients treated with focal brainstem radiotherapy (RT) relapse locally. There are several reports, which suggest that sub-lethal irradiation (IR) promotes migration of glioma cells (37-40). Wild-Bode et al. (37) proposed that tumor cells stimulated to invade by sub-lethal doses of irradiation may escape the target volume of post-operative RT, thereby evading delivery of a cumulative lethal dose and forming the basis for loco-regional relapse a few months after RT. These infiltrative tumors are less proliferative as shown by few mitoses and scarce MIB1 immunopositivity (12). Therefore, concurrent antiinvasive/anti-migratory therapies (cell cycle/check point inhibitors, glycogen synthase kinase-3 inhibitors: lithium chloride and 6-bromoindirubin-oxime) along with RT have been suggested to be beneficial in these patients potentially improving the efficacy of RT $(40,41)$.

The question of whether to offer focal vs. cranio-spinal RT in DIPG cannot be currently answered due to the paucity of data. Benesch et al. (30) in their series of HGG (including one patient with DIPG) did not observe a statistically significant difference in the progression free survival (PFS) and OS between patients with and without tumor dissemination at diagnosis. Hence, their recommendation was to treat both the groups in the same manner (focal IMRT). Similarly, data from the German registry (42) comparing six HGG patients who had metastasis at diagnosis (two patients with DIPG) and underwent intensified chemo-radiotherapy (CSI \pm local boost to tumor deposits with concurrent metronomic temozolomide) demonstrated inferior results when compared to the Benesch series (30). In the future, a multi-centered randomized clinical trial to address the issue of appropriate clinical target volume (CSI vs. focal RT) in DIPG may be considered. Although recurrence of tumor (either locally or at distant sites) has shorter OS, CNS prophylaxis in the form of regular intra-thecal chemotherapy (43) similar to acute lymphoblastic leukemia (ALL) therapy is not recommended.

\section{TREATMENT}

Diffuse intrinsic pontine glioma is almost invariably fatal with a mean OS of 8-14 months from the time of diagnosis. Radiation therapy (RT) prolongs survival by a mean of 3-6 months, but is still considered aggressive palliative therapy. A number of adjuvant therapies such as chemotherapy, targeted therapies, differentiation agents and radiation sensitizers have been studied, but none of them have had any significant impact on patient outcomes. There is no role for surgery in a typical case of DIPG. However, recent advances in understanding the biology of these tumors and availability of targeted therapies may warrant biopsy at diagnosis in some patients in order to personalize therapy and/ or enroll the child in a clinical trial.

\section{Radiation Therapy}

The current standard of care for newly diagnosed DIPG patients is fractionated focal intensity modulated radiation therapy (IMRT) 
to the tumor along with 1-2 cm margins (54-60 Gy, 1.8-2 Gy fractions, given once daily for 5 days per week over a period of 6 weeks). Supportive care in the form of corticosteroids is used to treat the peri-tumoral edema and is usually weaned after completion of RT. About $70-80 \%$ of patients respond to RT with some transient improvement in their neurological symptoms but more than half of the patients incur steroid induced side effects. Lower doses of RT (<50Gy) have worse outcomes, and higher doses using hyper-fractionated RT (66-78 Gy) do not appear to provide a survival advantage when compared to standard dose and fractionation protocols (44-54) (Table 1). It was postulated that re-population by rapidly proliferating tumor cells may be a reason for local treatment failure and accelerated fractionation (conventional fraction sizes twice daily) was expected to reduce the potential for re-population and improve outcomes. Conversely, several studies using hypo-fractionation have reported results that are similar to those observed with normal fractionation but offer no distinct survival benefits (52-54). Although the advantage of the hypofractionation technique is reduction in the total duration of treatment (13-18 fractions instead of 30-33), the major disadvantages are the adverse effects of RT on adjacent normal tissue. Hyperfractionation usually requires twice daily anesthetics in young children. Following completion of radiation, there is clinical progression of disease in almost all cases with radiologic evidence of local recurrence within 3-6 months. As discussed above, there could be LMD as well as distant parenchymal recurrence. Although RT appears beneficial, radiation sensitizers have not shown any improvement in outcomes to date. The use of RT concurrently with radio-sensitizers such as platinum compounds (carboplatin), topoisomerase inhibitors (etoposide, trofosfamide, topotecan), and other agents (metronomic temozolomide, etanidazole) has not been successful (Tables 1 and 3A,B).

\section{Feasibility of Palliative Re-Irradiation in DIPG}

There is evidence suggesting that re-irradiation in pediatric brain tumors (posterior fossa ependymoma, EP) is well tolerated and provides durable local tumor control $(55,56)$. Median time from first to second irradiation was 21.9 months (range, 7.5-67.7 months) (55) in one series and 26 months (range, 13-88 months) in another series (56). The median re-RT dose for focal fractionated re-irradiation (FFRT) was 55.2 Gy (range, 50.4-54 Gy) with a cumulative dose of 87-113 Gy to the brain stem and none of the patients had radiation necrosis (55).

Based on these studies in ependymoma and in the absence of effective treatment options for recurrent DIPG, some institutions are providing palliative re-irradiation with/without concurrent chemotherapy for DIPG $(57,58)$. Fontanilla et al. (57) treated six recurrent DIPG patients (clinical and MRI confirmation) with chemo-radiotherapy followed by post RT chemotherapy. The time to initial progression from the completion of first RT was 4 to 18 months and the time interval between initial RT and re-RT was 8-28 months. The re-RT dose was between 18 Gy $(n=1)$ to $20 \mathrm{~Gy}(n=4)$. There was good symptomatic response in four of the six patients with improvement in speech, swallowing and ambulation. The median clinical progression-free survival (PFS) time was 5 months and there were minimal RT related side effects with no Grade 3-4 toxicities reported. Massimino et al.
(58) re-irradiated 11 of their 16 locally relapsed DIPG patients. There was tumor volume shrinkage in 7 cases and steroids were weaned completely in 10 cases. The median survival after re-RT was 6 months (range, 6 weeks to 14 months) and none of the reirradiated patients showed any evidence of worsening symptoms or unexpected side effects. Preferred candidates for re-irradiation in DIPG may include those children who have experienced durable responses to prior radiation without evidence of clinical/ radiological progression off therapy $(57,59)$.

We analyzed data on 10 patients of recurrent DIPG treated with re-RT across different centers in Canada (Table 2; 60). Median time from diagnosis to progression was 12 months (range, 4-37 months) in this cohort, exceeding median time to progression in historical controls. Re-irradiation total dose varied between institutions from $21.6 \mathrm{~Gy}(n=2), 30.6 \mathrm{~Gy}$ $(n=6)$, and $36 \mathrm{~Gy}(n=2)$, administered in $1.8 \mathrm{~Gy}$ daily fractions. Re-radiation was to the involved field except in two patients who received whole brain irradiation due to distant/disseminated relapse. One patient received a third course of focal radiation $(21.6 \mathrm{~Gy}) 6$ months after re-radiation. Re-irradiation was tolerated very well by all patients. Four patients had transient fatigue and decreased appetite during treatment. All but one showed neurological improvement, with four patients showing full recovery. With a median follow-up from diagnosis of 19.5 months (range, 9-45 months), seven patients died, with a median time from re-irradiation to death of 9 months (range, 5-13 months). When compared to an historic cohort of 46 patients, median time from progression to death was 91.5 days in the non re-irradiated patients, vs. 171 days in the re-irradiated ones $(P<0.05)$. Although these preliminary results are encouraging, they are from small pilot studies and remain to be confirmed in a clinical trial setting. Although we do not suggest that re-irradiation should become the standard of care, based upon published and unpublished results, we encourage the development of prospective cooperative group Phase II studies offering re-irradiation so that this treatment option can be properly studied from a safety and efficacy perspective. Based on the above data, a dose range from 30 to $36 \mathrm{~Gy}$ in 16 to 20 fractions (1.8 Gy per fraction) at the time of recurrence may be a reasonable approach that is feasible and well tolerated.

\section{Chemotherapy}

Different chemotherapeutic strategies including high-dose myeloablative chemotherapy with stem cell rescue, neo-adjuvant (pre-RT) multi-agent chemotherapy, concurrent chemotherapy with RT (fractionated/hyper-fractionated protocols), and adjuvant chemotherapy have not demonstrated improved survival when compared to RT alone [Tables 3A,B (61-90)]. In particular, the current standard of care for adult GBM, namely RT with concurrent and adjuvant temozolomide, did not improve outcomes for DIPG [(91, 92), Table 4]. Various studies utilizing both neo-adjuvant and concurrent chemotherapies have shown no improvement in the OS of DIPG patients when compared to standard RT. Two French studies showed improved results using chemotherapy with RT when compared to RT alone. Doz et al. (65) used Carboplatin prior to and concurrently with RT in 38 DIPG patients and reported a median survival of 11 months 
TABLE 1 | Radiation therapy in DIPG.

\begin{tabular}{|c|c|c|c|c|c|c|}
\hline Reference & Year/phase & $\begin{array}{l}\text { Number of } \\
\text { patients }(\boldsymbol{N})\end{array}$ & $\begin{array}{l}\text { Treatment: total dose (Gy)/total } \\
\text { fractions/dose per fraction (Gy) }\end{array}$ & Imaging & Outcome & Comments \\
\hline Jenkin et al. (44) & 1987/RCT & 33 & $50-60 /-/ 0.8-0.9$ & CT & OS: $20 \%$ & $\begin{array}{l}\text { Patients randomized to RT with and without adjuvant } \\
\text { chemotherapy: CCNU, vincristine and prednisone } \\
\text { Procarbazine was given at relapse in patients who } \\
\text { received chemotherapy only } \\
\text { Brain stem gliomas (including DIPG) were included in the } \\
\text { study }\end{array}$ \\
\hline Packer et al. (45) & 1987/pilot & 16 & $64.8 / 54 / 1.2$ & CT/MRI & Median PFS: 7 months & \\
\hline $\begin{array}{l}\text { Freeman et al. } \\
(46)\end{array}$ & 1988/I-II & 34 & $66 / 60 / 1.1$ & CT & $\begin{array}{l}\text { MST: } 11 \text { months } \\
\text { OS at } 1 \text { year: } 48 \%\end{array}$ & $\begin{array}{l}\text { Brain stem gliomas (including DIPG) were included in the } \\
\text { study }\end{array}$ \\
\hline $\begin{array}{l}\text { Edwards et al. } \\
(47)\end{array}$ & 1989/I-II & 34 & $72 / 72 / 1$ & CT/MRI & $\begin{array}{l}\text { MST: } 16 \text { months } \\
\text { Median TTP: } 11 \text { months }\end{array}$ & $\begin{array}{l}\text { Brain stem gliomas (including DIPG) were included in the } \\
\text { study }\end{array}$ \\
\hline $\begin{array}{l}\text { Freeman et al. } \\
(48)\end{array}$ & 1991/I-II & 57 & $70.2 / 60 / 1.17$ & CT/MRI & $\begin{array}{l}\text { Median TTP: } 6 \text { months. MST: } 10 \text { months } \\
\text { OS at } 1 \text { year }-39.6 \pm 6.6 \% \text {, at } \\
2 \text { years }-23 \pm 5.8 \%\end{array}$ & \\
\hline Packer et al. (49) & 1993/-II & 53 & $72 / 72 / 1$ & CT/MRI & $\begin{array}{l}\text { OS at } 1 \text { year }-38 \pm 6.5 \% \text {, at } \\
2 \text { years }-14 \pm 5.4 \% \text {, at } 3 \text { years }-8 \pm 6.5\end{array}$ & \\
\hline $\begin{array}{l}\text { Freeman CR } \\
\text { et al. (27) }\end{array}$ & 1993/I-II & 39 & $75.6 / 60 / 1.26$ & $\mathrm{MRI}$ & $\begin{array}{l}\text { Median TTP: } 7 \text { months. MST: } 10 \text { months } \\
\text { OS at } 1 \text { year }-39.9 \pm 8.3 \% \text {, at } \\
2 \text { years }-7 \pm 4.8 \%\end{array}$ & \\
\hline Packer et al. (50) & 1994/I-II & 66 & $78 / 78 / 1$ & CT/MRI & OS at 1 year $-35 \pm 6 \%$, at 3 years $-11 \pm 6 \%$ & \\
\hline Packer et al. (51) & 1996/pilot & 32 & $72 / 72 / 1$ & MRI & Median TTP: 5 months & $\begin{array}{l}\text { Recombinant beta-interferon was used prior to and with } \\
\text { RT } \\
\text { Brain stem gliomas (including DIPG) were included in the } \\
\text { study }\end{array}$ \\
\hline Lewis et al. (52) & 1997/pilot & 28 & $\begin{array}{l}48.6 / 27 / 1.8 \\
50.4 / 28 / 1.8\end{array}$ & CT/MRI & $\begin{array}{l}\text { MST: } 8.5 \text { months } \\
\text { OS at } 1 \text { year }-32 \% \text {, at } 2 \text { years }-11 \%\end{array}$ & $\begin{array}{l}\text { Brain stem gliomas (including DIPG) were included in the } \\
\text { study } \\
\text { Biopsy was done in } 10 \text { cases with Grade I/II }(n=4) \text { and } \\
\text { Grade-III/IV }(n=6)\end{array}$ \\
\hline $\begin{array}{l}\text { Janssens et. } \\
\text { al. (53) }\end{array}$ & 2009/pilot & 9 & $\begin{array}{c}39 / 9 / 3(n=8) \\
33 / 6 / 5.5(n=1)\end{array}$ & $\mathrm{MRI}$ & $\begin{array}{l}\text { Median TTP: } 4.9 \text { months } \\
\text { OS: } 8.6 \text { months }\end{array}$ & Biopsy proven Grade IV astrocytomas in four patients \\
\hline $\begin{array}{l}\text { Negretti et.al. } \\
\text { (54) }\end{array}$ & $2011 /-$ & 22 & $49 / 15 / 3$ & $\mathrm{MRI}$ & $\begin{array}{l}\text { Median TTP: } 5.7 \text { months } \\
\text { OS: } 7.6 \text { months }\end{array}$ & \\
\hline
\end{tabular}

CT, computerized tomography; MRI, magnetic resonance imaging; OS, overall survival; PFS, progression free survival; MST, median survival time; TTP, time to tumor progression. 
TABLE 2 | Re-irradiation for DIPG (60).

\begin{tabular}{|c|c|c|c|c|c|c|c|c|c|c|}
\hline $\begin{array}{l}\text { Patient/ } \\
\text { sex }\end{array}$ & Age at Dx & $\begin{array}{l}\text { Time from Dx to } \\
\text { Relapse (m) }\end{array}$ & $\begin{array}{l}\text { Time from } \\
\text { Relapse to start } \\
\text { re-RT (d) }\end{array}$ & $\begin{array}{l}\text { Dose of } \\
\text { re-RT } \\
\text { (Gy) }\end{array}$ & $\begin{array}{l}\text { MRI post } \\
\text { re-RT }\end{array}$ & DXM & $\begin{array}{l}\text { Clinical (neurologic } \\
\text { symptoms) } \\
\text { improvement after re-RT }\end{array}$ & $\begin{array}{l}\text { Side effects after } \\
\text { re-RT }\end{array}$ & $\begin{array}{l}\text { Time from } \\
\text { re-RT to progression } \\
(\mathrm{m})\end{array}$ & $\begin{array}{l}\text { Outcome/ } \\
\text { follow up (m) }\end{array}$ \\
\hline $1 / F$ & $\begin{array}{l}6 \text { years } 5 \\
\text { months }^{\mathrm{a}}\end{array}$ & 14 & 20 & 30.6/focal & Yes & $\begin{array}{l}\text { Yes/weaned by end } \\
\text { of RT }\end{array}$ & Yes/partial recovery & Fatigue, insomnia & 6 & Dead (25) \\
\hline $2 / F$ & $\begin{array}{l}10 \text { years } \\
3 \text { months }\end{array}$ & 4 & 12 & 30.6/focal & - & $\begin{array}{l}\text { Yes/weaned at } \\
\text { progression }\end{array}$ & No & $\begin{array}{l}\text { Vomiting, fatigue, } \\
\text { weakness }\end{array}$ & 5 & Dead (9) \\
\hline $3 / F$ & 5 years & 9 & 14 & 30.6/focal & Yes & $\begin{array}{l}\text { Yes/weaned by end } \\
\text { of RT }\end{array}$ & Yes/fully recovered & None & 3 & Dead (15) \\
\hline $4 / \mathrm{M}$ & 13 years $^{b}$ & $9^{c}$ & 24 & $\begin{array}{l}30.6 / \text { whole } \\
\text { brain }\end{array}$ & Yes & Nil & Yes/fully recovered & None & 12 & Alive (37) \\
\hline $5 / F$ & $\begin{array}{l}4 \text { years } \\
9 \text { months }{ }^{d}\end{array}$ & $32^{c}$ & 75 & $\begin{array}{l}\text { 30.6/whole } \\
\text { brain }\end{array}$ & Yes & Nil & Yes/fully recovered & $\begin{array}{l}\text { Fatigue, } \\
\text { decreased energy, } \\
\text { appetite }\end{array}$ & 7 & Dead (45) \\
\hline 6/M & $\begin{array}{l}2 \text { years } \\
3 \text { months }\end{array}$ & 13 & 40 & 30.6/focal & - & Nil & Yes/fully recovered & None & - & Alive (16) \\
\hline $7 / F$ & 5 years & $10^{f}$ & 60 & 21.6/focal & Yes & Nil & No & None & - & Dead (7) \\
\hline 8/M & $\begin{array}{l}4 \text { years } \\
6 \text { months }^{9}\end{array}$ & 6 & 15 & 21.6/focal & Yes & Nil & Yes/fully recovered & None & - & Dead (4) \\
\hline 9/F & 5 years $^{h}$ & 36 & 30 & 36/focal & Yes & Nil & Yes/partial recovery & None & 3 & Dead (4) \\
\hline $10 / \mathrm{M}$ & 9 years $^{\text {h }}$ & 12 & 30 & 36/focal & Yes & Yes & Yes/partial recovery & Fatigue & 9 & Dead (9) \\
\hline
\end{tabular}

D, days; Dx, diagnosis; Gy, Gray.

aThis patient had a second relapse 6 months after re-RT and received a third course of re-RT with 21.6 Gy.

${ }^{b}$ Started on Temozolomide 3 months after re-RT and continued until progression 1 year after re-RT.

cLeptomeningeal/parenchymal dissemination at recurrence.

${ }^{d}$ Atypical presentation at diagnosis: MRI showed expansive brain stem lesion centered in the pons, and a second non-enhancing lesion in the right cerebellar hemisphere. The lesions were biopsied revealing an anaplastic astrocytoma. EFavorable prognostic factors at diagnosis: age <3 years and prolonged interval between onset of symptoms and diagnosis (10 months). Biopsy at diagnosis revealed glioblastoma multiforme.

'Received Bevacizumab (Avastin), biweekly, at the time of relapse for a total of eight doses.

sProlonged interval between onset of symptoms and diagnosis (14 months). The child had an alteration of voice with no other clinical symptoms.

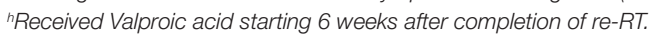


TABLE 3 | (A) Summary of clinical trials of neo-adjuvant chemotherapy and RT in DIPG; (B) summary of clinical trials of Chemotherapy (pre-/concurrent/adjuvant) and RT in DIPG.

\begin{tabular}{|c|c|c|c|c|c|}
\hline Reference & Year/phase & $\begin{array}{l}\text { Number of } \\
\text { patients }(n)\end{array}$ & Treatment & Outcome & RT and comments \\
\hline $\begin{array}{l}\text { Kretschmar et al. (61) } \\
\text { POG-8833 }\end{array}$ & 1993/II & 32 & $\begin{array}{l}\text { CDDP/CPM - four cycles followed by } \\
\text { RT }\end{array}$ & MST: 9 months & RT dose: 66/60/1.1 \\
\hline Dunkel et al. (62) & 1998/pilot & 6 & $\begin{array}{l}\text { BCNU/Etop/Te followed by ABMT and } \\
\text { RT }\end{array}$ & MST: $11.4(7.6-17.1)$ & RT dose: 72-78/72-78/1 \\
\hline Jakacki et al. (63) & 1999/II & 11 & $\begin{array}{l}\text { CCNU/PNCR followed by ABMT and } \\
\text { RT }\end{array}$ & MST: 13 months & RT dose: 50.4-59.4/-/1.8 \\
\hline $\begin{array}{l}\text { Jennings et al. (64) } \\
\text { CCG-9941 }\end{array}$ & 2002/II & $\begin{array}{l}\text { Group } A=32 \\
\text { Group } B=31\end{array}$ & $\begin{array}{l}\mathrm{A}=\mathrm{VCR} / \mathrm{Car} / \text { Etop } \\
\mathrm{B}=\mathrm{CDDP} / \mathrm{CPM} / \text { Etop/VCR followed } \\
\text { by RT }\end{array}$ & $\begin{array}{l}\text { EFS at } 1 \text { year }-17 \pm 5 \% \\
2 \text { years }-6 \pm 3 \%\end{array}$ & RT dose: 72/72/1 \\
\hline Doz et al. (65) & 2002/pilot & 38 & Car followed by Car + RT & MST: 11 months & $\begin{array}{l}\text { RT dose: 54/30/1.8 } \\
\text { Biopsy in } 14 \text { patients (HGG-7, LGG-4) }\end{array}$ \\
\hline $\begin{array}{l}\text { Grundy et al. } \\
\text { (66) UKCCSG/ } \\
\text { SIOP-CNS9204 }\end{array}$ & 2010/II & 7 & $\begin{array}{l}\text { Car/CDDP/CPM/HDMTxNCR followed } \\
\text { by RT }\end{array}$ & $\begin{array}{l}\text { Median TTP: } 0.21 \text { years }(0.1-0.53) \\
\text { Median OS: } 0.3 \text { years }(0.25-1.43)\end{array}$ & \\
\hline $\begin{array}{l}\text { Frappaz et.al. (67), } \\
\text { SFOP-BSG-98 }\end{array}$ & 2008/II & 23 & $\begin{array}{l}\text { BCNU/CDDP/Hydroxyurea/HDMTX/ } \\
\text { Tamoxifen followed by RT }\end{array}$ & MST: 17 months (10-23) & $\begin{array}{l}\text { RT dose: } 54 / 2 / 2 \\
\text { Prolonged hospitalization in treated patients }\end{array}$ \\
\hline Massimino et al. (68) & 2008/II, Study-II & 10 & $\begin{array}{l}\text { CDDP/CPM/Etop/HDMTxNCR } \\
\text { followed by RT }\end{array}$ & $\begin{array}{l}\text { MST: } 13 \text { months } \\
\text { OS } 1 \text { year }-70 \pm 14 \% \text {, } \\
2 \text { years }-10 \pm 9 \%\end{array}$ & $\begin{array}{l}\text { All patients received adjuvant CCNUNCR chemotherapy post } \\
\text { RT }\end{array}$ \\
\hline $\begin{array}{l}\text { Wolff et al. (69) } \\
\text { (HIT-GBM-D) }\end{array}$ & 2011/II & 7 & $\begin{array}{l}\text { HDMTx (two doses) followed by } \\
\text { RT + CDDP/Etop/lfos/NCR }\end{array}$ & Median EFS: 0.55 & $\begin{array}{l}\text { All patients received adjuvant CCNU/NCR/Prednisone } \\
\text { chemotherapy post RT }\end{array}$ \\
\hline Levin et al. (70) & 1984/II & 28 & CCNU/5FU followed by RT & MST: 11 months & $\begin{array}{l}\text { RT dose: } 55 / 31 / 1.8 \\
\text { Patients received adjuvant hydroxyurea/misonidazole post RT } \\
\text { Brain stem gliomas (including DIPG) were included in the study }\end{array}$ \\
\hline Wakabayashi et al. (71) & 1992/Pilot & 16 & $\mathrm{RT}+\mathrm{ACNU/FN- \beta}$ & MST: 15.7 months & $\begin{array}{l}\text { RT dose: } 40-60 / 27-30 / 1.5-2 \\
\text { Brain stem gliomas (including DIPG) were included in the study }\end{array}$ \\
\hline Bouffet et al. (72) & 1997/II & 8 & BCNU-ABMT followed by RT & MST: 4 months & $\begin{array}{l}\text { Patients were previously treated with Chemo/RT } \\
\text { Brain stem gliomas (including DIPG) were included in the study }\end{array}$ \\
\hline Walter et al. (73) & 1998/I-II & 9 & RT + Car/Etop & $\begin{array}{l}\text { Median TTP: } 8 \text { months } \\
\text { OS: } 1 \text { year }-44 \%, 2 \text { years }-11 \%\end{array}$ & RT dose: 70.2/60/1.7 \\
\hline Allen et al. (74) & 1999/I-II & 31 & $\mathrm{RT}+\mathrm{Car}$ & Median OS: 12 months & $\begin{array}{l}\text { RT dose: 72/72/1 } \\
\text { Three long-term survivors (mean F/U } 79 \text { months) }\end{array}$ \\
\hline Jakacki et al. (63) & 1999/pilot-II & 6 & $\begin{array}{l}\text { CCNU/PNCR + ABMT (pre-/during/ } \\
\text { post) RT }\end{array}$ & MST: 11 months & RT dose: 50.4-59.4/28-33/1.8 \\
\hline Mandell LR et al. (75) & $\begin{array}{l}\text { 1999/III } \\
\text { (randomized) }\end{array}$ & $\begin{array}{l}\text { Group-I }=66 \\
\text { Group-II }=64\end{array}$ & $\mathrm{RT}+\mathrm{CDDP}$ & $\begin{array}{l}\text { Median TTP: } 6 \text { months (I)/5 months (II) } \\
\text { OS } 1 \text { year }-30.9(\mathrm{I}) / 27(\mathrm{II}), 2 \text { years }-7.1 \\
\text { (I)/6.7(II) }\end{array}$ & $\begin{array}{l}\text { RT dose: Group-l: 54/30/1.8. Group-II: 70.2/60/1.17 } \\
\text { This study showed that hyperfractionation has no benefit } \\
\text { compared to routine fractionation }\end{array}$ \\
\hline
\end{tabular}


TABLE 3 | Continued

\begin{tabular}{|c|c|c|c|c|c|}
\hline Reference & Year/phase & $\begin{array}{l}\text { Number of } \\
\text { patients }(n)\end{array}$ & Treatment & Outcome & RT and comments \\
\hline $\begin{array}{l}\text { Freeman et al. (76), } \\
\text { POG-9239 }\end{array}$ & $2000 / 11$ & 64 & RT with and without CDDP & $\begin{array}{l}1 \text { year survival lower in CDDP group } \\
\text { compared to RT alone }\end{array}$ & $\begin{array}{l}\text { RT dose: } 70.2 / 60 / 1.7 \\
\text { Brain stem gliomas (including DIPG) were included in the study }\end{array}$ \\
\hline Bouffet et al. (77) & 2000/Pilot & 36 & RT followed by Bu/Te and ABMT & MST: 10 months & $\begin{array}{l}\text { RT dose: } 54 / 30 / 1.8 \\
\text { Brain stem gliomas (including DIPG) were included in the study }\end{array}$ \\
\hline Broniscer et al. (78) & 2000/II & 27 & $\mathrm{RT}+$ tamoxifen followed by Tamoxifen & OS 1 year- $37 \pm 9.5 \%$ & RT dose: 54-60/34-36/1.5-1.8 \\
\hline $\begin{array}{l}\text { Wolff et al. (79), } \\
\text { HIT-GBM-A }\end{array}$ & 2002/pilot & 20 & $\mathrm{RT}+$ trophosphamide/Etop & OS 1 year- $0.4,4$ years- 0.05 & $\begin{array}{l}\text { RT dose: 54/30/1.8 } \\
\text { Biopsy in } 15 \text { patients (Grade II-4, III-3, IV-8) }\end{array}$ \\
\hline Marcus et al. (80) & 2003/1 & 18 & $\mathrm{RT}+$ Etanidazole & MST: 8.5 months (3-58) & RT dose: 66/44/1.5 \\
\hline Sanghavi et al. (81) & $2003 / 1$ & 16 & RT + Topotecan & $\begin{array}{l}\text { MST: } 15 \text { months }(9.6-19) \\
\text { OS } 1 \text { year } 53 \%\end{array}$ & RT dose: 59.4/33/1.8 \\
\hline $\begin{array}{l}\text { Bernier-Chastagnier } \\
\text { et al. (82) }\end{array}$ & $2005 / 11$ & 32 & RT + Topotecan & MST: 8.3 months OS 1 year $25.5 \pm 8 \%$ & RT dose: 54/30/1.8 \\
\hline Packer et al. (83) & $2005 / 1$ & 13 & Car + RMP-7 followed by RT & MST: 11 months & $\begin{array}{l}\text { RT dose: } 59.4 / 33 / 1.8 \\
\text { RMP- } 7 \text { (cereport) is a synthetic bradykinin analog that increases } \\
\text { BBB permeability }\end{array}$ \\
\hline Greenberg et al. (84) & $2005 / 1$ & 7 & RT + Etop/NCR/cyclosporine-A & MST: 11 months & $\begin{array}{l}\text { RT dose: 54/30/1.8 } \\
\text { Cyclosporine was used for P-gp inhibition }\end{array}$ \\
\hline $\begin{array}{l}\text { Wolff et al. (85), } \\
\text { HIT-GBM-B }\end{array}$ & 2006/I-II & 19 & $\begin{array}{l}\mathrm{RT}+\mathrm{CDDP} / \text { Etop/lfos followed by } \\
\mathrm{CPM} / \mathrm{FN}-\gamma\end{array}$ & MST: 9.6 months & RT dose: 54/30/1.8 \\
\hline Warren et al. (86) & $2006 / 11$ & 12 & RMP-7 + Car & No response in any patient & Brain stem gliomas (including DIPG) were included in the study \\
\hline Turner et al. (87) & $2007 / \|$ & 12 & $\begin{array}{l}\text { RT + Thalidomide followed by } \\
\text { Thalidomide }\end{array}$ & $\begin{array}{l}\text { Median TTP: } 5 \text { months (2-11) } \\
\text { Median TTD: } 9 \text { months (5-17) }\end{array}$ & RT dose: 55.8/31/1.8 \\
\hline Korones et al. (88) & 2008/II & 30 & $\begin{array}{l}\text { RT }+ \text { VCR/Etop (oral) followed by VCR/ } \\
\text { Etop (oral) } \times 10 \text { cycles }\end{array}$ & $\begin{array}{l}\text { MST: } 9 \text { months }(3-36) \text {, OS } \\
1 \text { year }-27 \pm 7 \%, 2 \text { year }-3 \pm 2 \%\end{array}$ & RT dose: 54/30/1.8 \\
\hline Massimino et al. (68) & $\begin{array}{l}\text { 2008/II, study-I } \\
\text { Study-III } \\
\text { Study-IV }\end{array}$ & $\begin{array}{l}21 \\
17 \\
14\end{array}$ & $\begin{array}{l}\mathrm{RT}+\text { Ara-C/Act-D/CDDP/Etop/lfos } \\
\mathrm{RT}+\mathrm{CDDP} \text { Etop/isotretinoin (prior/ } \\
\text { during and after RT) } \\
\mathrm{RT}+\text { vinorelbine (prior/during and after } \\
\mathrm{RT} \text { ) }\end{array}$ & $\begin{array}{l}\text { MST: } 12 \text { months, OS } 1 \text { year }-45 \pm 6 \% \text {, } \\
2 \text { year }-18 \pm 5 \% \\
\text { MST: } 9 \text { months, OS } 1 \text { year }-29 \pm 11 \% \text {, } \\
2 \text { year }-12 \pm 8 \% \\
\text { MST: } 9 \text { months, OS } 1 \text { year }-43 \pm 13 \% \text {, } \\
2 \text { year }-21 \pm 11 \%\end{array}$ & $\begin{array}{l}\text { RT dose: 54/30/1.8 }(n=18), 66 / 60 / 1.1(n=3) \\
\text { RT dose: 54-60/27-30/1.8-2 } \\
\text { RT dose: 54-60/27-30/2 }\end{array}$ \\
\hline Michalski et al. (89) & 2009/II & 31 & RT + tamoxifen (oral) & MST: 6.32 months, OS 1 year $-16.1 \%$ & RT dose: 54/30/1.8 \\
\hline $\begin{array}{l}\text { Wolff et al. (90), } \\
\text { HIT-GBM-C }\end{array}$ & 2010/II & 37 & $\begin{array}{l}\text { RT + CDDP/Etop/NCR followed by } \\
\text { CDDP/Etop/lfos/valproic acid }\end{array}$ & Median OS: $1.13(0.87-1.39)$ & RT dose: 54/30/1.8 \\
\hline
\end{tabular}


TABLE 4 | Concomitant and Adjuvant Temozolomide (TMZ) based chemotherapy in DIPG.

\begin{tabular}{|c|c|c|c|c|c|}
\hline Reference & Year/phase & $\begin{array}{l}\text { Number of } \\
\text { patients }(N)\end{array}$ & $\begin{array}{l}\text { TMZ dose }\left(\mathrm{mg} / \mathrm{m}^{2}\right) \text { with } \mathrm{RT} / \\
\text { after } \mathrm{RT} / \mathrm{number} \text { of cycles }\end{array}$ & Outcome & Comments \\
\hline $\begin{array}{l}\text { Lashford } \\
\text { et al. (93) }\end{array}$ & $2002 / 11$ & 21 & -/200/- & MST: 6.2 months & $\begin{array}{l}\text { All patients received } \mathrm{RT} \\
\mathrm{TMZ} \text { started at relapse }\end{array}$ \\
\hline $\begin{array}{l}\text { Broniscer et al. } \\
(94)\end{array}$ & 2005/II & 33 & $-/ 200 / 6$ & $\begin{array}{l}\text { MST: } 12 \text { months } \\
\text { OS: } 1 \text { year }-48 \%\end{array}$ & $\begin{array}{l}\text { All patients received irinotecan and } R T \\
\text { RT dosing: } 50.4-70.8 / 30 / 1.8\end{array}$ \\
\hline $\begin{array}{l}\text { Sirachainan } \\
\text { et al. (95) }\end{array}$ & 2008/II & 12 & 75/200/- & $\begin{array}{l}\text { Median TTP: } 10.2 \pm 3 \text { months } \\
\text { MST: } 13.5 \pm 3.6 \text { months } \\
\text { OS: } 1 \text { year }-58 \pm 4.2 \%\end{array}$ & $\begin{array}{l}\text { All patients received cis-retinoic acid. } \\
\text { RT dose: } 35.8-59.4 \text { Gy }\end{array}$ \\
\hline Jalali et al. (96) & 2010/II & 20 & $75 / 200 / 12$ & $\begin{array}{l}\text { Median OS: } 9.15 \text { months } \\
\text { Median PFS: } 6.9 \text { months }\end{array}$ & RT dose: 54/30/1.8 \\
\hline Chiang et al. (97) & 2010/II & $\begin{array}{l}I=10 \\
I I=8\end{array}$ & $75 / 150 /-$ & $\begin{array}{l}\text { MST: } 12.3 \text { months } \\
\text { OS: } 1 \text { year }-51 \%\end{array}$ & $\begin{array}{l}\mathrm{I}=\text { adjuvant } \mathrm{TMZ} \\
\mathrm{II}=\text { Concurrent } \mathrm{TMZ}\end{array}$ \\
\hline Kim et al. (98) & $2010 / 1-\|$ & 12 & $75 / 200 /-$ & $\begin{array}{l}\text { MST: } 12.7 \text { months } \\
\text { OS: } 1 \text { year }-58 \%\end{array}$ & All patients received thalidomide with $\mathrm{RT}$ \\
\hline Sharp et al. (99) & 2010/II & 15 & 85/85/- & $\begin{array}{l}\text { MST: } 9.8 \text { months } \\
\text { OS: } 1 \text { year }-20 \%\end{array}$ & RT dose: 54/30/1.8 \\
\hline $\begin{array}{l}\text { Cohen et al. (91) } \\
\text { ACNS0126 }\end{array}$ & $2011 / 11$ & 63 & $90 / 200 / 10$ & $\begin{array}{l}\text { MST: } 9 \text { months } \\
\text { OS: } 1 \text { year }-40 \%, 2 \text { year }-3.6 \%\end{array}$ & RT dose: 59.4/33/1.8 \\
\hline $\begin{array}{l}\text { Chassot } \\
\text { et al. (92) }\end{array}$ & 2012/II & 21 & $75 / 200 / 6$ & $\begin{array}{l}\text { MST: } 11.7 \text { months } \\
\text { OS: } 1 \text { year }-50 \%\end{array}$ & $\begin{array}{l}\text { RT dose: } 54 / 30 / 1.8 \\
\text { All cases were biopsied }\end{array}$ \\
\hline $\begin{array}{l}\text { Kebudi } \\
\text { et al. (100) }\end{array}$ & $\begin{array}{l}\text { 2012/ } \\
\text { retrospective }\end{array}$ & 21 & $75 / 200 / 12$ & $\begin{array}{l}\text { MST: } 12 \text { months } \\
\text { OS: } 1 \text { year }-55 \%, 2 \text { year }-37 \%\end{array}$ & \\
\hline $\begin{array}{l}\text { Bailey } \\
\text { et al. (101) }\end{array}$ & 2013/II & 43 & $75 / 75-100 / 12$ & $\begin{array}{l}\text { MST: } 9.5 \text { months } \\
\text { OS: } 1 \text { year }-35 \%, 2 \text { year }-17 \%\end{array}$ & \\
\hline Zaky et al. (102) & $\begin{array}{l}\text { 2013/ } \\
\text { retrospective }\end{array}$ & 6 & $75 / 200 /-$ & $\begin{array}{l}\text { Median EFS: } 6.1 \text { months. } \\
\text { Median OS: } 9.6 \text { months }\end{array}$ & $\begin{array}{l}\text { Patients received concurrent chemo } \\
\text { (carboplatin/etoposide) followed by } \\
\text { adjuvant chemo (irinotecan/bevacizumab) }\end{array}$ \\
\hline $\begin{array}{l}\text { Aguilera } \\
\text { et al. (103) }\end{array}$ & 2013/report & 2 & $-/ 200 /-$ & PFS: $37 / 47$ months & $\begin{array}{l}\text { RT dose: } 54 / 30 / 1.8 \\
\text { Both patients received adjuvant } \\
\text { bevacizumab and were alive at the time of } \\
\text { reporting } \\
\text { Tumor size decreased by }>65 \%\end{array}$ \\
\hline $\begin{array}{l}\text { Muller } \\
\text { et al. (42) }\end{array}$ & $2014 / I 1$ & 2 & $75 /-1-$ & MST: 11.9/8.1 months & $\begin{array}{l}\text { Both patients received nimotuzumab and } \\
\text { cranio-spinal RT }\end{array}$ \\
\hline $\begin{array}{l}\text { Rizzo } \\
\text { et al. (104) }\end{array}$ & 2015/II & 14 & $75 / 200 / 12$ & Median TTP: 7.15 months & \\
\hline
\end{tabular}

OS, overall survival; PFS, progression free survival; MST, median survival time; TTP, time to progression; EFS, event free survival; RT, radiation therapy; RT dose, cumulative dose (Gy)/number of fractions/dose per fraction (Gy).

(compared to 9 months with RT alone). Frappaz et al. (67) used frontline chemotherapy (Tamoxifen/BCNU/Cisplatin alternating with high dose methotrexate/HDMTX) aimed at delaying radiation until time of clinical progression. Median survival increased significantly in patients participating in the protocol compared to historical controls ( 17 vs. 9 months, $P=0.022$ ). However there was prolonged hospitalization and increased infections in the chemotherapy group. The German HIT-GBM protocols assessed a variety of chemotherapeutic strategies in the HIT-GBM protocols $(\mathrm{A}-\mathrm{D})$ in the treatment of DIPG and could not demonstrate any survival benefit when compared to RT alone $(69,79,85,90)$. In a single institution study over two decades Massimino et al. (68), evaluated four different regimes in the treatment of DIPG: (1) intensive high-dose courses of chemotherapy (cisplatin/ etoposide, cyclophosphamide/vincristine/methotrexate) and a subsequent course of myeloablative thiotepa followed by RT and maintenance chemotherapy; (2) cisplatin/etoposide followed by isotretinoin before, during, and after focal RT; (3) concurrent chemo-radiotherapy (etoposide, cytarabine, ifosfamide, cisplatin, and dactinomycin); and (4) Vinorelbine before, during, and after radiotherapy. The median survival time was reported as 11 months with no survival advantage within protocols compared with RT alone (Tables 3A,B).

Various clinical trials using Temozolomide (TMZ) in combination with concurrent RT followed by adjuvant TMZ have shown variable results (91-104) (Table 4). Kebudi et al. (100) in their retrospective analysis of 50 DIPG patients treated with RT and TMZ with and without other chemotherapy showed 
significantly higher OS in the TMZ treated group. Patients receiving $\mathrm{RT}+\mathrm{TMZ}+$ chemotherapy (other than TMZ) showed prolonged survival when compared to patients receiving only RT $(P=0.005)$. However a number of other studies (Table 4) have reported no distinct survival advantage of RT + TMZ when compared to RT alone. The HIT-HGG-2007 study is a noninferiority clinical trial designed to prove the hypothesis that ambulatory TMZ-chemoRT is as effective, but less toxic than the previous HIT-GBM (C-D) trials (42). At the present time, adjuvant chemotherapy is not recommended for DIPG outside of a clinical trial.

\section{Impediments to Chemotherapy/Targeted Therapy}

One of the major limitations of DIPG treatment is the successful and efficient delivery of effective therapies to the tumor. For a drug or a targeted therapeutic agent to be effective, the active compound: (a) should reach its target in the tumor cells, (b) in adequate concentrations/bind to the target present in the tumor cells, (c) for an adequate amount of time for tumor cytotoxicity, and (d) the tumor cells themselves should be sensitive to the drug/ agent. There are several factors that can affect drug concentrations within the DIPG tumor (105). These include bio-availability of the drug/agent (serum levels, protein/tissue binding), rate of blood flow to the tumor, degree of brain penetration [blood-brain barrier (BBB) and blood tumor barrier] and drug metabolism. The BBB is frequently intact in DIPG and plays an active role in restricting the delivery of systemically administered conventional and biological therapies (106). This often leads to decreased effective concentration of the therapeutic agents in the tumor.

In order to overcome the restrictive ability of the $\mathrm{BBB}$, several alternative drug delivery strategies have been tried including: transient osmotic disruption of the $\mathrm{BBB}$, enhancing the lipophilicity of drugs, inhibition of membrane efflux pumps, intra-arterial/ intra-thecal chemotherapy, intra-nasal chemotherapy, direct intra-tumoral chemotherapy, etc., but each has met with minimal success (107). The most clinically proven approach to increase drug penetration into the CNS is to reversibly disrupt the junctions formed by the endothelial cells to enhance their penetration through intercellular junctions. This can be accomplished through the use of osmotic agents or pharmacologically through the targeting of membrane receptors that alter BBB permeability. While osmotic agents such as mannitol have been used to modulate BBB permeability in both pre-clinical and clinical settings (107) the major drawback with osmotic disruption is the long recovery period required for reestablishment of BBB integrity (108). With several hours required for the return of normal BBB function, patients are susceptible to toxicity (109). On the other hand, pharmacological agents such as RMP-7/Cereport, a bradykinin receptor agonist, have been shown to transiently disrupt the $\mathrm{BBB}$ in various animal models $(108,110)$. However, these agents have failed to produce the desired response in clinical trials due to non-uniform disruption of the $\mathrm{BBB}(83,86,110)$. Veringa et al. (106) using patient derived cell cultures and tumors showed that DIPG cells express MRP1 and the tumor vasculature exhibits drug efflux transporters (P-gp, MRP1, and BCRP1). However, cell cultures were sensitive to an array of chemotherapeutic agents when treated in vitro. The concurrent use of efflux inhibitors (111) along with chemotherapy might improve the efficacy of chemotherapy and could be considered in a prospective clinical trial. Convection-enhanced delivery (CED), using external or implantable subcutaneous pumps, allows intra-tumoral injection of novel therapeutic agents (chemotherapy, cytotoxic interleukins, radio-immunotherapeutic agents), and there are ongoing phase I/ II clinical trials in DIPG.

\section{Biological Targeted Therapy}

Due to the availability of tumor tissue (from both autopsy and surgical biopsy samples) in several centers across the world, novel targets have been identified in DIPG and there are several ongoing

\begin{tabular}{|c|c|c|c|c|c|}
\hline Target & Agent & $\begin{array}{l}\text { Newly diagnosed or progressive/relapsed, } \\
\text { number of patients }(n)\end{array}$ & $\begin{array}{l}\text { Median PFS } \\
\text { (months) }\end{array}$ & $\begin{array}{l}\text { PFS at } \\
6 \text { months (\%) }\end{array}$ & Reference \\
\hline \multirow[t]{5}{*}{ EGFR } & Erlotinib & Newly diagnosed with RT & 8 & 90 & Geoerger et al. (115) \\
\hline & Gefitinib & Newly diagnosed with RT $(n=43)$ & 7.4 & 88 & Pollack et al. (116) \\
\hline & & Newly diagnosed with RT $(n=20)$ & NR & 48 & Geyer et al. (117) \\
\hline & Nimotuzumab & Newly diagnosed with RT $(n=41)$ & 5.5 & NR & Massimino et al. (118) \\
\hline & & Progressive/relapsed $(n=44)$ & 1.7 & NR & Bartels et al. (119) \\
\hline EGFR/NEGFR & Vandetanib & Newly diagnosed with RT $(n=21)$ & NR & 88 & Broniscer et al. (120) \\
\hline PDGFRA & Imatinib & Newly diagnosed with RT & NR & 70 & Pollack et al. (121) \\
\hline \multirow[t]{3}{*}{ VEGF } & Bevacizumab & Progressive/relapsed $(n=31)$ & 2.3 & 9.7 & Gururangan et al. (122) \\
\hline & & Progressive/relapsed $(n=2)$ & 2.25 & NR & Narayana et al. (123) \\
\hline & & Newly diagnosed with RT $(n=14)$ & 8.8 & NR & Salloum et al. (124) \\
\hline mTOR & Temsirolimus & Progressive/relapsed $(n=5)$ & 2.5 & NR & Geoerger et al. (125) \\
\hline Farnesyl transferase & Tipifarnib & Newly diagnosed with RT $(n=40)$ & NR & 44 & Haas-Kogan et al. (126) \\
\hline Integrins ( $\alpha \vee \beta 3$ and $\alpha \vee \beta 5)$ & Cilengitide & Progressive/relapsed $(n=31)$ & NR & NR & MacDonald et al. (127) \\
\hline Histone de-acetylase & Valproic acid & Newly diagnosed with RT/chemotherapy & 9.5 & NR & Felix et al. (128) \\
\hline (HDAC) & Panabinostat & Murine DIPG models & - & - & Grasso et al. (129) \\
\hline
\end{tabular}

PFS, progression free survival; RT, radiation therapy; NR, not recorded. 
clinical trials using targeted therapies. The reader is referred to excellent reviews summarizing the past and ongoing clinical trials involving targeted therapies in DIPG (112-114). However, none of these trials have shown any superior survival benefit when compared to RT alone (Table 5) (115-129).

\section{Immunological/Vaccine Therapy}

Immunotherapy in DIPG is limited due to the non-availability of tumor tissue to identify and generate tumor-associated antigens. Li et al. (130) evaluated the expression of Epidermal Growth Factor Receptor variant III (EGFRvIII) in postmortem DIPG tissue and primary cell cultures using various laboratory techniques (immunohistochemistry/IHC, western blot, RT-PCR, and flow cytometry). IHC revealed expression of EGFRvIII in four of nine cases and the staining pattern was similar to the adult tumors. Overall expression was detected in 6 of 11 cases. There are several ongoing phase III clinical trials using a peptide vaccine targeting EGFRvIII in the treatment of newly diagnosed GBM in adults. Zhou et al. (131) evaluated the expression of B7-H3, a membrane protein (CD276) against which a therapeutic monoclonal antibody is available (8H9). IHC revealed B7-H3 immunoreactivity in $100 \%(n=9 / 9)$ of postmortem DIPG samples.

Pollack et al. (132) conducted a pilot study using a peptidebased vaccine against novel GAA [Glioma associated antigens - EphA2, interleukin-13 receptor alpha2 (IL-13R $\alpha 2$ ), and survivin]-derived epitopes in children with DIPG and HGGs. Newly diagnosed DIPG patients [treated with RT alone $(N=14)$ and RT + chemotherapy $(N=8)]$ were administered the vaccine in conjunction with immuno-adjuvant polyinosinic-polycytidylic acid (poly [I: C]) stabilized by lysine and carboxymethylcellulose (poly-ICLC). Five children had symptomatic pseudo-progression, which responded to dexamethasone and was associated with prolonged survival. The vaccine was well tolerated overall with good clinical (OS between 6.3 to $>38$ months) and immunological response (positive anti-GAA immune response in 8 patients). Based upon the feasibility, safety, and potential promise of these studies, immune-based therapeutic strategies are expected to be offered in prospective phase II and III clinical trials.

\section{PROGNOSTIC/BIOLOGIC MARKERS IN DIPG}

Although the outcome of DIPG is extremely poor and uniformly fatal, there are a sub-group of patients who survive longer. Some of the favorable clinical prognostic factors are young age at diagnosis ( $<3$ years), prolonged interval between onset of symptoms and diagnosis ( $>6$ months) and absence of cranial nerve palsies or long tract involvement at presentation. Tools to assess the efficacy of treatment and monitoring in DIPG are evolving. The RANO (Response Assessment in Neuro-oncology) criteria (using MRI) are used to assess response during or post chemotherapy/targeted therapy with decrease in steroid use, tumor size and improved neurologic symptoms indicative of response to therapy. However, DIPG tumors frequently do not enhance or may exhibit variable patterns of enhancement and either decrease in tumor size is not sustained or this does not translate into improved survival (133). Moreover, conventional MRI instructs us regarding tumor structure and location and cannot reliably differentiate therapyrelated phenomena such as efficacy, pseudo-progression, or pseudo-response (133). Hipp et al. (134) used prospective multiparametric imaging to evaluate outcome in children with DIPG. In their study, increased perfusion [using dynamic susceptibility contrast (DSC) MRI] at any single time point was associated with shorter survival $(\mathrm{RR}=4.91)$, and increasing perfusion over time was a poor prognostic factor. Steffen-Smith et al. (135) used Magnetic Resonance Spectroscopy (MRS) techniques to monitor DIPG patients throughout their course of treatment. In their study, the choline: $N$-acetylaspartate ratio (CHO:NAA) was shown to be prognostic; patients with a CHO:NAA ratio higher than the median of 2.1 demonstrated a greater risk of early mortality compared to patients with a ratio of $\leq 2.1$. During follow-up, changes in this ratio had an impact on prognosis with increase in the CHO: NAA ratio being inversely associated with survival and decreasing CHO: NAA ratio being associated with a longer life expectancy. Although very promising, these imaging modalities are limited by their availability in specialized centers and only as part of research protocols.

Diffuse intrinsic pontine glioma patients who have histone mutations (H3.3 and H3.1, K27M) identified by IHC and other methods have a worse OS when compared to wild-type tumors, and this association is independent of patient age and histological diagnosis. Jansen et al. (136) recently developed a DIPG survival prediction tool that can be used for predicting outcome and risk stratification for prospective future clinical trials. Multivariate Cox proportional hazards analysis was used to pick both positive prognostic predictors (longer symptom duration, age $\leq 3$ years and use of concurrent oral and intravenous chemotherapy with RT) and negative predictor (presence of ring enhancement in contrast MRI). The risk score calculated based on these predictors will not only able to predict the prognosis but also stratify patients into risk groups (standard, intermediate, and high), which can be used in future clinical trials. This prediction model itself requires validation in a prospective clinical trial.

\section{SUMMARY}

In summary, diagnostic and therapeutic failures of the past and recent advances in the genomics and biology of DIPG have made us wiser in the following ways: (1) we now understand that DIPG is a heterogeneous group of tumors with origins that can be traced to aberrant neurodevelopment and it is biologically distinct from other pediatric and adult HGG. Therefore, adapting chemotherapy and/or targeted therapy used in pediatric and adult HGG for DIPG should be abandoned. (2) biopsy of DIPG is relatively safe using modern neurosurgical techniques and should be considered in the context of clinical trials. Biopsy results will confirm the diagnosis, may identify actionable targets, and with the use of appropriate prediction tools, help us to risk-stratify these patients for both therapy and prognosis. (3) there is evidence in the literature to suggest that DIPG is a whole brain disease and this may support whole neuraxis imaging at diagnosis, during therapy and at follow-up. (4) previous decades of treatment failure may be attributed to several factors, including: inadequate drug levels within the tumor due to lack of $\mathrm{BBB}$ 
permeability, single agent testing, signaling pathway redundancy leading to drug resistance, lack of patient pre-selection, lack of biomarkers to assess response and off-target effects. In the future, judicious use of radiation may also include appropriate concurrent/adjuvant therapies directed against signaling pathways, genetic or epigenetic targets; $\mathrm{BBB}$ permeability agents and/or anti-invasive/anti-migration agents might improve the efficacy of these combined treatment modalities. (5) Pre-clinical testing and validation of appropriately chosen targets [e.g., JMJD3, H3K27 demethylase (137), Menin (138), ACVR1 (24)] in available tumor models $(138,139)$ will help to identify novel effective therapies and provide options to personalize treatment and limit side effects and long-term toxicities. Expedited clinical trials using these targets in small numbers of patients in tertiary centers will facilitate the development of larger multicenter randomized clinical trials. Use of validated biomarkers (140) along with

\section{REFERENCES}

1. Ostrom QT, de Blank PM, Kruchko C, Petersen CM, Liao P, Finlay JL, et al. CBTRUS statistical report: ALSF infant and childhood primary brain and central nervous system tumors diagnosed in the United States in 2007-2011. Neuro Oncol (2015) 17(Suppl 1):v1-35. doi:10.1093/neuonc/nou327

2. Epstein F. A staging system for brainstem gliomas. Cancer (1985) 56:1804-6.

3. Recinos PF, Sciubba DM, Jallo GI. Brainstem tumors: where are we today? Pediatr Neurosurg (2007) 43:192-201. doi:10.1159/000098831

4. Choux M, Lena G, Do L. Brainstem tumors. In: Choux M, Di Rocco C, Hockley A, editors. Pediatric Neurosurgery. New York, NY: Churchill Livingstone (2000). p. 471-91.

5. Albright A, Packer R, Zimmerman R, Rorke L, Boyett J, Hammond G. Magnetic resonance scans should replace biopsies for the diagnosis of diffuse brainstem gliomas: a report from the children's cancer group. Neurosurgery (1993) 33:1026-9. doi:10.1227/00006123-199312000-00010

6. Cartmill M, Punt J. Diffuse brain stem glioma. A review of stereotactic biopsies. Childs Nerv Syst (1999) 15:235-7. doi:10.1007/s003810050379

7. Roujeau T, Machado G, Garnett MR, Miquel C, Puget S, Geoerger B, et al. Stereotactic biopsy of diffuse pontine lesions in children. J Neurosurg (2007) 107(1 Suppl):1-4.

8. Cage TA, Samagh SP, Mueller S, Nicolaides T, Haas-Kogan D, Prados M, et al. Feasibility, safety, and indications for surgical biopsy of intrinsic brainstem tumors in children. Childs Nerv Syst (2013) 29:1313-9. doi:10.1007/ s00381-013-2101-0

9. Wang ZJ, Rao L, Bhambhani K, Miller K, Poulik J, Altinok D, et al. Diffuse intrinsic pontine glioma biopsy: a single institution experience. Pediatr Blood Cancer (2015) 62:163-5. doi:10.1002/pbc.25224

10. Pirotte B, Lubansu A, Massager N, Wikler D, Goldman S, Levivier M. Results of positron emission tomography guidance and reassessment of the utility of and indications for stereotactic biopsy in children with infiltrative brainstem tumors. J Neurosurg (2007) 107:392-9. doi:10.3171/PED-07/11/392

11. Chen X, Weigel D, Ganslandt O, Buchfelder M, Nimsky C. Diffusion tensor imaging and white mater tractography in patients with brainstem lesions. Acta Neurochir (2007) 149:1117-31. doi:10.1007/s00701-007-1282-2

12. Buczkowicz P, Bartels U, Bouffet E, Becher O, Hawkins C. Histopathological spectrum of pediatric diffuse intrinsic pontine glioma: diagnostic and therapeutic implications. Acta Neuropathol (2014) 128:573-81. doi:10.1007/ s00401-014-1319-6

13. Puget $\mathrm{S}$, Blauwblomme T, Grill J. Is biopsy safe in children with newly diagnosed diffuse intrinsic pontine glioma? Am Soc Clin Oncol Educ Book (2012) 32:629-33. doi:10.14694/EdBook_AM.2012.32.629

14. Corti M, Trione N, Risso D, Soto I, Villafañe MF, Yampolsky C, et al. Disseminated paracoccidioidomycosis with a single brainstem lesion. A case report and literature review. Neuroradiol J (2010) 23(4):454-8. doi:10.1177/197140091002300416

15. Barkovich AJ, Krischer J, Kun LE, Packer R, Zimmerman RA, Freeman CR, et al. Brain stem gliomas: a classification system based on magnetic resonance imaging. Pediatr Neurosurg (1990-1991) 16(2):73-83. appropriate neuraxis (brain and spine) and advanced imaging techniques (DSC-MRI, MRS) could assist us to monitor patients with DIPG for therapy response and toward earlier detection of relapse/progression. The time has come for the pediatric neurooncology community to unite in their efforts through the rapid integration of data from basic science and translational research along with appropriately conducted clinical trials to conquer this deadly disease.

\section{ACKNOWLEDGMENTS}

Dr. Issai Vanan is a Scholar at the Father Peter J. Mckenna St. Baldrick's Cancer Research, supported by the St. Baldrick's Foundation (USA). Dr. Eisenstat holds the Muriel and Ada Hole Kids with Cancer Society Chair in Pediatric Oncology, University of Alberta (Canada).

16. Fischbein NJ, Prados MD, Wara W, Russon C, Edwards MS, Barkovich AJ. Radiologic classification of brain stem tumors: correlation of magnetic resonance imaging appearance with clinical outcome. Pediatr Neurosurg (1996) 24(1):9-23. doi:10.1159/000121010

17. Donaldson SS, Laningham F, Fisher PG. Advances toward an understanding of brainstem gliomas. J Clin Oncol (2006) 24:1266-72. doi:10.1200/ JCO.2005.04.6599

18. Hankinson TC, Campagna EJ, Foreman NK, Handler MH. Interpretation of magnetic resonance images in diffuse intrinsic pontine glioma: a survey of pediatric neurosurgeons. J Neurosurg Pediatr (2011) 8:97-102. doi:10.3171/2011.4.PEDS1180

19. Kieran MW. Time to rethink the unthinkable: upfront biopsy of children with newly diagnosed diffuse intrinsic pontine glioma (DIPG). Pediatr Blood Cancer (2015) 62:3-4. doi:10.1002/pbc.25266

20. Fontebasso AM, Papillon-Cavanagh S, Schwartzentruber J, Nikbakht H, Gerges N, Fiset PO, et al. Recurrent somatic mutations in ACVR1 in pediatric midline high-grade astrocytoma. Nat Genet (2014) 46(5):462-6. doi:10.1038/ ng.2950

21. Venneti S, Santi M, Felicella MM, Yarilin D, Phillips JJ, Sullivan LM, et al. A sensitive and specific histopathologic prognostic marker for H3F3A K27M mutant pediatric glioblastomas. Acta Neuropathol (2014) 128(5):743-53. doi:10.1007/s00401-014-1338-3

22. Bechet D, Gielen GGH, Korshunov A, Pfister SM, Rousso C, Faury D, et al. Specific detection of methionine 27 mutation in histone 3 variants (H3K27M) in fixed tissue from high-grade astrocytomas. Acta Neuropathol (2014) 128(5):733-41. doi:10.1007/s00401-014-1337-4

23. Khuong-Quang D, Buczkowicz P, Rakopoulos P, Liu XY, Fontebasso AM, Bouffet E, et al. K27M mutation in histone H3.3 defines clinically and biologically distinct subgroups of pediatric diffuse intrinsic pontine gliomas. Acta Neuropathol (2012) 124:439-47. doi:10.1007/s00401-012-0998-0

24. Buczkowicz P, Hoeman C, Rakopoulus P, Pajovic S, Letourneau L, Dzamba $\mathrm{M}$, et al. Genomic analysis of diffuse intrinsic pontine gliomas identifies three molecular subgroups and recurrent activating ACVR1 mutations. Nat Genet (2014) 46(5):451-6. doi:10.1038/ng.2936

25. Mantravadi RV, Phatak R, Bellur S, Liebner EJ, Hass R. Brain stem gliomas: an autopsy study of 25 cases. Cancer (1982) 49(6):1294-6. doi:10.1002/10970142(19820315)49:6<1294::AID-CNCR2820490636>3.0.CO;2-V

26. Halperin EC. Pediatric brain stem tumors: patterns of treatment failure and their implications for radiotherapy. Int J Radiat Oncol Biol Phys (1985) 11(7):1293-8. doi:10.1016/0360-3016(85)90244-5

27. Freeman CR, Krischer JP, Sanford A, Cohen ME, Burger PC, Carpio RD, et al. Final results of a study of escalating doses of hyperfractionated radiotherapy in brain stem tumors in children: a pediatric oncology group study. Int J Radiat Oncol Biol Phys (1993) 27(2):197-206. doi:10.1016/0360-3016(93)90228-N

28. Donahue B, Allen J, Siffert J, Rosovsky M, Pinto R. Patterns of recurrence in brain stem gliomas: evidence for cranio-spinal dissemination. Int J Radiat Oncol Biol Phys (1998) 40(3):677-80. doi:10.1016/ S0360-3016(97)00846-8 
29. Yoshimura J, Onda K, Tanaka R, Takahashi H. Clinico-pathological study of diffuse type brainstem gliomas: analysis of 40 autopsy cases. Neurol Med Chir (Tokyo) (2003) 43:375-82. doi:10.2176/nmc.43.375

30. Benesch M, Wagner S, Berthold F, Wolff JEA. Primary dissemination of high-grade gliomas in children: experiences from four studies of the pediatric oncology and hematology society of the german language group (GPOH). $J$ Neurooncol (2005) 72:179-83. doi:10.1007/s11060-004-3546-5

31. Wagner S, Benesch M, Berthold F, Gnekow AK, Rutkowski S, Strater R, et al. Secondary dissemination in children with high-grade malignant gliomas and diffuse intrinsic pontine gliomas. Br J Cancer (2006) 95:991-7. doi:10.1038/ sj.bjc. 6603402

32. Gururangan S, McLaughlin CA, Brashears J, Watral MA, Provenzale J, Coleman E, et al. Incidence and patterns of neuraxis metastases in children with diffuse pontine glioma. J Neurooncol (2006) 77:207-12. doi:10.1007/ s11060-005-9029-5

33. Sethi R, Allen J, Donahue B, Karajannis M, Gardner S, Wisoff J, et al. Prospective neuraxis MRI surveillance reveals a high risk of leptomeningeal dissemination in diffuse intrinsic pontine glioma. J Neurooncol (2011) 102:121-7. doi:10.1007/s11060-010-0301-y

34. Caretti V, Jansen MHA, van Vuurden DG, Lagerweij R, Bugiani M, Horsman I, et al. Implementation of a multi-institutional diffuse intrinsic pontine glioma autopsy protocol and characterization of a primary cell culture. Neuropathol Appl Neurobiol (2013) 39:426-36. doi:10.1111/j.1365-2990.2012.01294.x

35. Caretti V, Bugiani M, Freret M, Schellen P, Jansen M, van Vuurden DG, et al. Subventricular spread of diffuse intrinsic pontine glioma. Acta Neuropathol (2014) 128(4):605-7. doi:10.1007/s00401-014-1307-x

36. Vanan MI, Eisenstat DD. Management of high grade gliomas in the pediatric patient: (past), present and the future. Neurooncol Pract (2014) 1(4):145-57. doi:10.1093/nop/npu022

37. Wild-Bode C, Weller M, Rimner A, Dichgans J, Wick W. Sub lethal irradiation promotes migration and invasiveness of glioma cells: implications for radiotherapy of human glioblastoma. Cancer Res (2001) 61(6):2744-50.

38. Zhai GG, Malhotra R, Delaney M, Latham D, Nestler U, Zhang M, et al. Radiation enhances the invasive potential of primary glioblastoma cells via activation of the Rho signaling pathway. J Neurooncol (2006) 76:227-37. doi:10.1007/s11060-005-6499-4

39. Park MC, Park MJ, Kwak HJ, Lee HC, Kim MS, Lee SH, et al. Ionizing radiation enhances matrix metalloproteinase- 2 secretion and invasion of glioma cells through Src/epidermal growth factor receptor-mediated p38/Akt and phosphatidylinositol 3-kinase/Akt signaling pathways. Cancer Res (2006) 66(17):8511-9. doi:10.1158/0008-5472.CAN-05-4340

40. Vanan I, Dong Z, Tosti E, Warshaw G, Symons M, Ruggieri R. Role of DNA damage checkpoint pathway in ionizing radiation-induced glioblastoma cell migration and invasion. Cell Mol Neurobiol (2012) 32:1199-208. doi:10.1007/ s10571-012-9846-y

41. Cockle JV, Picton S, Levesley J, Ilett E, Carcaboso AM, Short S, et al. Cell migration in pediatric glioma; characterization and potential therapeutic targeting. Br J Cancer (2015) 112(4):693-703. doi:10.1038/bjc.2015.16

42. Muller K, Schlamann A, Guckenberger M, Warmuth-Metz M, Gluck A, Pietschmann S, et al. Craniospinal irradiation with concurrent temozolomide for primary metastatic pediatric high-grade or diffuse intrinsic pontine gliomas. Strahlenther Onkol (2014) 190:377-81. doi:10.1007/s00066-013-0513-0

43. Slavc I, Schuller E, Falger G, Gunes M, Pillwein K, Czech T, et al. Feasibility of long-term intraventricular therapy with mafosfamide $(n=26)$ and etoposide $(\mathrm{n}=11)$ : experience in 26 children with disseminated malignant brain tumors. J Neurooncol (2003) 64(3):239-47. doi:10.1023/A:1025633704071

44. Jenkin RDT, Boesel C, Ertel I, Evans A, Hittle R, Ortega J, et al. Brain-stem tumors in childhood: a prospective randomized trial of irradiation with and without adjuvant CCNU, VCR and prednisone. A report of the childrens cancer group study. J Neurosurg (1987) 66(2):227-33. doi:10.3171/ jns.1987.66.2.0227

45. Packer RJ, Littman PA, Sposto RM, D’Angio G, Priest JR, Heideman RL, et al. Results of a pilot study of hyper-fractionated radiation therapy for children with brain stem gliomas. Int J Radiat Oncol Biol Phys (1987) 13:1647-51. doi:10.1016/0360-3016(87)90160-X

46. Freeman CR, Krischer J, Sanford RA, Burger PC, Cohen M, Norris D, et al. Hyper-fractionated radiotherapy in brain stem tumors: results of a pediatric oncology group study. Int J Radiat Oncol Biol Phys (1988) 15(2):311-8. doi:10.1016/S0360-3016(98)90010-4
47. Edwards MSB, Wara WM, Urtasun RC, Prados M, Levin VA, Fulton D, et al. Hyper-fractionated radiation therapy for brain-stem glioma: a phase I-II trial. J Neurosurg (1989) 70(5):691-700. doi:10.3171/jns.1989.70.5.0691

48. Freeman CR, Krischer J, Sanford RA, Cohen M, Burger PC, Kun L, et al. Hyper-fractionated radiation therapy in brain stem tumors: results of treatment at the 7020 cGy dose level of pediatric oncology group study \#8495. Cancer (1991) 68:474-81. doi:10.1002/1097-0142(19910801)68:3<474::AIDCNCR2820680305>3.0.CO;2-7

49. Packer RJ, Boyett JM, Zimmerman RA, Rorke LB, Kaplan AM, Albright $\mathrm{AL}$, et al. Hyper-fractionated radiation therapy (72Gy) for children with brain stem gliomas. Cancer (1993) 72:1414-21. doi:10.1002/10970142(19930815)72:4<1414::AID-CNCR2820720442>3.0.CO;2-C

50. Packer RJ, Boyett JM, Zimmerman RA, Albright AL, Kaplan AM, Rorke $\mathrm{LB}$, et al. Outcome of children with brain stem gliomas after treatment with 7800cGy of hyper-fractionated radiotherapy. Cancer (1994) 74:1827-34. doi:10.1002/1097-0142(19940915)74:6<1827::AID-CNCR2820740628>3.0.CO;2-Q

51. Packer RJ, Prados M, Phillips P, Nicholson HS, Boyett JM, Goldwein J, et al. Treatment of children with newly diagnosed brain stem gliomas with intravenous recombinant beta-interferon and hyper-fractionated radiation therapy. Cancer (1996) 77(10):2150-6. doi:10.1002/ (SICI) 1097-0142(19960515)77:10<2150::AID-CNCR28>3.0.CO;2-T

52. Lewis J, Lucraft H, Gholkar A. UKCCSG study of accelerated radiotherapy for pediatric brain stem gliomas. Int J Radiat Oncol Biol Phys (1997) 38(5):925-9. doi:10.1016/S0360-3016(97)00134-X

53. Janssens GRJ, Gidding CEM, Van Lindert EJ, Oldenburger FR, Erasmus CE, Schouten-Meeteren AYN, et al. The role of hypo-fractionation radiotherapy for diffuse intrinsic brainstem glioma in children: a pilot study. Int J Radiat Oncol Biol Phys (2009) 73(3):722-6. doi:10.1016/j.ijrobp.2008.05.030

54. Negretti L, Bouchireb K, Levy-Piedbois C, Habrand JL, Dhermain F, Kalifa C, et al. Hypo-fractionated radiotherapy in the treatment of diffuse intrinsic pontine glioma in children: a single institution's experience. J Neurooncol (2011) 104:773-7. doi:10.1007/s11060-011-0542-4

55. Merchant TE, Boop FA, Kun LE, Sanford RA. A retrospective study of surgery and re-irradiation for recurrent ependymoma. Int J Radiat Oncol Biol Phys (2008) 71(1):87-97. doi:10.1016/j.ijrobp.2007.09.037

56. Bouffet E, Hawkins CE, Ballourah W, Taylor MD, Bartels UK, Schoenhoff $\mathrm{N}$, et al. Survival benefit for pediatric patients with recurrent ependymoma treated with re-irradiation. Int J Radiat Oncol Biol Phys (2012) 83(5):1541-8. doi:10.1016/j.ijrobp.2011.10.039

57. Fontanilla HP, Pinnix CC, Ketonen LM, Woo SY, Vats TS, Rytting ME, et al. Palliative re-irradiation for progressive diffuse intrinsic pontine glioma. Am J Clin Oncol (2012) 35:51-7. doi:10.1007/s00381-013-2350-y

58. Massimino M, Biassoni V, Miceli R, Schiavello E, Warmuth-Metz M, Modena $P$, et al. Results of nimotuzumab and vinorelbine, radiation and re-irradiation for diffuse intrinsic pontine glioma. J Neurooncol (2014) 118:305-12. doi:10.1007/s11060-014-1428-z

59. Khatua S, Hou P, Bodiwala R, Wolff J, Hamilton J, Patil S, et al. Preliminary experience with diffusion tensor imaging before and after re-irradiation treatments in children with progressive diffuse pontine glioma. Childs Nerv Syst (2014) 30:925-30. doi:10.1007/s00381-013-2350-y

60. Lassaletta $\mathrm{A}$, Bartels $\mathrm{U}$, Strother $\mathrm{D}$, Issaivanan $\mathrm{M}, \mathrm{Ku} \mathrm{D}$, Laperriere $\mathrm{N}$, et al. Re-Irradiation in patients with Diffuse Intrinsic Pontine Gliomas-The Canadian Experience. Poster Presentation-SIOP 2015, Cape Town, South Africa (2015).

61. Kretschmar CS, Tarbell NJ, Barnes PD, Krischer JP, Burger PC, Kun L. Preirradiation chemotherapy and hyper-fractionated radiation therapy (66 Gy) for children with brain stem tumors. A phase II study of the pediatric oncology group, protocol 8833. Cancer (1993) 72(4):1404-13. doi:10.1002/10970142(19930815)72:4<1404::AID-CNCR2820720441>3.0.CO;2-G

62. Dunkel IJ, Garvin JH, Goldman S, Ettinger LJ, Kaplan AM, Cairo M, et al. High dose chemotherapy with autologous bone marrow rescue for children with diffuse pontine brain stem tumors. children's cancer group. J Neurooncol (1998) 37(1):67-73. doi:10.1023/A:1005874508975

63. Jakacki RI, Siffert J, Jamison C, Velasquez L, Allen JC. Dose-intensive, time-compressed procarbazine, CCNU, vincristine (PCV) with peripheral blood stem cell support and concurrent radiation in patients with newly diagnosed high-grade gliomas. J Neurooncol (1999) 44(1):77-83. doi:10.10 23/A:1006360222643 
64. Jennings MT, Sposto R, Boyett JM, Vezina LG, Holmes E, Berger MS, et al. Pre-radiation chemotherapy in primary high-risk brainstem tumors: phase II study CCG-9941 of the children's cancer group. J Clin Oncol (2002) 20(16):3431-7. doi:10.1200/JCO.2002.04.109

65. Doz F, Neuenschwander S, Bouffet E, Gentet JC, Schneider P, Kalifa C, et al. Carboplatin before and during radiation therapy for the treatment of malignant brain stem tumours: a study by the Socie'te' Franc, aise d'Oncologie Pe'diatrique. Eur J Cancer (2002) 38:815-9. doi:10.1016/ S0959-8049(02)00029-1

66. Grundy RG, Wilne SH, Robinson KJ, Ironside JW, Cox T, Chong WK, et al. Primary postoperative chemotherapy without radiotherapy for treatment of brain tumours other than ependymoma in children under 3 years: results of the first UKCCSG/SIOP CNS 9204 trial. Eur J Cancer (2010) 46(1):120-33. doi:10.1016/j.ejca.2009.09.013

67. Frappaz D, Schell M, Thiesse P, Marec-Berard P, Mottolese C, Bergeron C, et al. Pre-radiation chemotherapy may improve survival in pediatric diffuse intrinsic brainstem gliomas: final results of BSG 98 prospective trial. Neuro Oncol (2008) 10(4):599-607. doi:10.1215/15228517-2008-029

68. Massimino M, Spreafico F, Biassoni V, Simonetti F, Riva D, Trecate G, et al. Diffuse pontine gliomas in children: changing strategies, changing results? A mono-institutional 20-year experience. J Neurooncol (2008) 87:355-61. doi:10.1007/s11060-008-9525-5

69. Wolff JE, Kortmann RD, Wolff B, Pietsch T, Peters O, Schmid HJ, et al. High dose methotrexate for pediatric high grade glioma-results of the HIT-GBM-D pilot study. J Neurooncol (2011) 102:433-42. doi:10.1007/s11060-010-0334-2

70. Levin VA, Edwards MS, Wara WM, Allen J, Ortega J, Vestnys P. 5-Fluorouracil and CCNU followed by hydroxyurea, misonidazole and irradiation for brain stem gliomas: a pilot study of the brain tumor research center and the childrens cancer group. Neurosurgery (1984) 14(6):679-81. doi:10.1227/00006123-198406000-00006

71. Wakabayashi T, Yoshida J, Mizuno M, Kito A, Sugita K. Effectiveness of interferon-B, ACNU and radiation therapy in pediatric patients with brainstem glioma. Neurol Med Chir (Tokyo) (1992) 32:942-6. doi:10.2176/nmc.32.942

72. Bouffet E, Khelfaoui F, Philip I, Biron P, Brunat-Mentigny M, Philip T. Highdose carmustine for high-grade gliomas in childhood. Cancer Chemother Pharmocol (1997) 39:376-9. doi:10.1007/s002800050586

73. Walter AW, Gajjar A, Ochs JS, Langston JW, Sanford RA, Kun LE, et al. Carboplatin and etoposide with hyper-fractionated radiotherapy in children with newly diagnosed diffuse pontine gliomas: a phase I/II study. Med Pediatr Oncol (1998) 30:28-33. doi:10.1002/ (SICI) 1096-911X(199801)30:1<28::AID-MPO9>3.0.CO;2-2

74. Allen J, Siffert J, Donahue B, Nirenberg A, Jakacki R, Robertson P, et al. A phase I/II study of carboplatin combined with hyper-fractionated radiotherapy for brainstem gliomas. Cancer (1999) 86:1064-9. doi:10.1002/ (SICI) 1097-0142(19990915)86:6<1064::AID-CNCR24>3.0.CO;2-1

75. Mandell LR, Kadota R, Freeman C, Douglass EC, Fontanesi J, Cohen ME, et al. There is no role for hyper-fractionated radiotherapy in the management of children with newly diagnosed diffuse intrinsic brainstem tumors: results of a pediatric oncology group phase III trial comparing conventional vs. hyper-fractionated radiotherapy. Int J Radiat Oncol Biol Phys (1999) 43(5):959-64. doi:10.1016/S0360-3016(98)00501-X

76. Freeman CR, Kepner J, Kun LE, Sanford RA, Kadota R, Mandell L, et al. A detrimental effect of a combined chemotherapy-radiotherapy approach in children with diffuse intrinsic brain stem gliomas? Int J Radiat Oncol Biol Phys (2000) 47(3):561-4. doi:10.1016/S0360-3016(00)00471-5

77. Bouffet E, Raquin M, Doz F, Gentet JC, Rodary C, Demeocq F, et al. Radiotherapy followed by high dose busulfan and thiotepa: a prospective assessment of high dose chemotherapy in children with diffuse pontine gliomas. Cancer (2000) 88:685-92. doi:10.1002/ (SICI) 1097-0142(20000201)88:3<685::AID-CNCR27>3.0.CO;2-K

78. Broniscer A, da Costa Leite C, Lanchote VL, Machado TMS, Cristofani LM. Radiation therapy and high-dose tamoxifen in the treatment of patients with diffuse brainstem gliomas: results of a Brazilian cooperative study. J Clin Oncol (2000) 18(6):1246-53.

79. Wolff JE, Westphal S, Molenkamp G, Gnekow A, Warmuth-Metz M, Rating $\mathrm{D}$, et al. Treatment of pediatric pontine glioma with oral trophosphamide and etoposide. Br J Cancer (2002) 87:945-9. doi:10.1038/sj.bjc.6600552

80. Marcus KJ, Dutton SC, Barnes P, Coleman CN, Pomeroy SL, Goumnerova $\mathrm{L}$, et al. A phase I trial of etanidazole and hyperfractionated radiotherapy in children with diffuse brainstem glioma. Int J Radiat Oncol Biol Phys (2003) 55(5):1182-5. doi:10.1016/S0360-3016(02)04391-2

81. Sanghavi SN, Needle MN, Krailo MD, Geyer JR, Ater J, Mehta MP. A phase I study of topotecan as a radiosensitizer for brainstem glioma of childhood: first report of the children's cancer group-0952. Neuro Oncol (2003) 5(1):8-13. doi:10.1093/neuonc/5.1.8

82. Bernier-Chastagner V, Grill J, Doz F, Bracard S, Gentet JC, Marie-Cardine A, et al. Topotecan as a radiosensitizer in the treatment of children with malignant diffuse brainstem gliomas: results of a French society of paediatric oncology phase II study. Cancer (2005) 104:2792-7. doi:10.1002/cncr.21534

83. Packer RJ, Krailo M, Mehta M, Warren K, Allen J, Jakacki R, et al. Phase 1 study of concurrent RMP-7 and carboplatin with radiotherapy for children with newly diagnosed brainstem gliomas. Cancer (2005) 104:1281-7. doi:10.1002/cncr.21301

84. Greenberg ML, Fisher PG, Freeman C, Korones DN, Bernstein M, Friedman $\mathrm{H}$, et al. Etoposide, vincristine, and cyclosporin A with standard-dose radiation therapy in newly diagnosed diffuse intrinsic brainstem gliomas: a pediatric oncology group phase I study. Pediatr Blood Cancer (2005) 45(5):644-8. doi:10.1002/pbc.20382

85. Wolff JE, Wagner S, Reinert C, Gnekow A, Kortmann RD, Kuhl J, et al. Maintenance treatment with interferon-gamma and low dose cyclophosphamide for pediatric high-grade glioma. J Neurooncol (2006) 79(3):315-21. doi:10.1007/s11060-006-9147-8

86. Warren K, Jakacki RI, Widemann B, Aikin A, Libucha M, Packer RJ, et al. Phase II trial of intravenous lobradimil and carboplatin in childhood brain tumors: a report from the children's oncology group. Cancer Chemother Pharmacol (2006) 58:343-7. doi:10.1007/s00280-005-0172-7

87. Turner CD, Chi S, Marcus KJ, MacDonald T, Packer RJ, Poussaint TY, et al. Phase II study of thalidomide and radiation in children with newly diagnosed brain stem gliomas and glioblastoma multiforme. J Neurooncol (2007) 82:95-101. doi:10.1007/s11060-006-9251-9

88. Korones DN, Fisher PG, Kretschmar C, Zhou T, Chen Z, Kepner J, et al. Treatment of children with diffuse intrinsic brain stem glioma with radiotherapy, vincristine and oral VP-16: a children's oncology group phase II study. Pediatr Blood Cancer (2008) 50(2):227-30. doi:10.1002/pbc.21154

89. Michalski A, Bouffet E, Taylor RE, Hargrave D, Walker D, Picton S, et al. The addition of high-dose tamoxifen to standard radiotherapy does not improve the survival of patients with diffuse intrinsic pontine glioma. J Neurooncol (2010) 100(1):81-8. doi:10.1007/s11060-010-0141-9

90. Wolff JE, Driever PH, Erdlenbruch B, Kortmann RD, Rutkowski S, Pietsch $\mathrm{T}$, et al. Intensive chemotherapy improves survival in pediatric high-grade glioma after gross total resection: results of the HIT-GBM-C protocol. Cancer (2010) 116(3):705-12. doi:10.1002/cncr.24730

91. Cohen K, Heideman R, Zhou T, Holmes EJ, Lavey RS, Bouffet E, et al. Temozolomide in the treatment of children with newly diagnosed diffuse intrinsic pontine gliomas: a report from the children's oncology group. Neuro Oncol (2011) 4:410-6. doi:10.1093/neuonc/noq205

92. Chassot A, Canale S, Varlet P, Puget S, Roujeau T, Negretti L, et al. Radiotherapy with concurrent and adjuvant temozolomide in children with newly diagnosed diffuse intrinsic pontine glioma. J Neurooncol (2012) 106:399-407. doi:10.1007/s11060-011-0681-7

93. Lashford LS, Thiesse P, Jouvet A, Jaspan T, Couanet D, Griffiths PD, et al. Temozolomide in malignant gliomas of childhood: a United Kingdom children's cancer study group and French society for pediatric oncology intergroup study. J Clin Oncol (2002) 20:4684-91. doi:10.1200/ JCO.2002.08.141

94. Broniscer A, Iacono L, Chintagumpala M, Fouladi M, Wallace D, Bowers DC, et al. Role of temozolomide after radiotherapy for newly diagnosed diffuse brainstem glioma in children. Cancer (2005) 103:133-9. doi:10.1002/ cncr.20741

95. Sirachainan N, Pakakasama S, Visudithbhan A, Chiamchanya S, Tuntiyatorn L, Dhanachai $\mathrm{M}$, et al. Concurrent radiotherapy with temozolomide followed by adjuvant temozolomide and cis-retinoic acid in children with diffuse intrinsic pontine glioma. Neuro Oncol (2008) 10:577-82. doi:10.1215/15228517-2008-025

96. Jalali R, Raut N, Arora B, Gupta T, Dutta D, Munshi A, et al. Prospective evaluation of radiotherapy with concurrent and adjuvant temozolomide in children with newly diagnosed diffuse intrinsic pontine glioma. Int J Radiat Oncol Biol Phys (2010) 77:113-8. doi:10.1016/j.ijrobp.2009.04.031 
97. Chiang KL, Chang KP, Lee YY, Huang PI, Hsu TR, Chen YW, et al. Role of temozolomide in the treatment of newly diagnosed diffuse brainstem glioma in children: experience at a single institution. Childs Nerv Syst (2010) 26:1035-41. doi:10.1007/s00381-010-1106-1

98. Kim CY, Kim SK, Phi JH, Lee MM, Kim IA, Kim IH, et al. A prospective study of temozolomide plus thalidomide during and after radiation therapy for pediatric diffuse pontine gliomas: preliminary results of the Korean society for pediatric neuro-oncology study. J Neurooncol (2010) 100:193-8. doi:10.1007/s11060-010-0157-1

99. Sharp JR, Bouffet E, Stempak D, Gammon J, Stephens D, Johnston DL, et al. A multi-centre Canadian pilot study of metronomic temozolomide combined with radiotherapy for newly diagnosed pediatric brainstem glioma. Eur J Cancer (2010) 46:3271-9. doi:10.1016/j.ejca.2010.06.115

100. Kebudi R, Cakir FB, Yaman Agaoglu F, Gorgun O, Ayan I, Darendeliler E. Pediatric diffuse intrinsic pontine glioma patients from a single center. Childs Nerv Syst (2013) 29(4):583-8. doi:10.1007/s00381-012-1986-3

101. Bailey S, Howman A, Wheatley K, Wherton D, Boota N, Pizer B, et al. Diffuse intrinsic pontine glioma treated with prolonged temozolomide and radiotherapy - results of a United Kingdom phase II trial (CNS-2007-04). Eur J Cancer (2013) 49:3856-62. doi:10.1016/j.ejca.2013.08.006

102. Zaky W, Wellner M, Brown RJ, Bluml S, Finlay JL, Dhall G. Treatment of children with diffuse intrinsic pontine gliomas with chemoradiotherapy followed by a combination of temozolomide, irinotecan and bevacizumab. Pediatr Hematol Oncol (2013) 30(7):623-32. doi:10.3109/08880018.2013.82 9895

103. Aguilera DG, Mazewski C, Hayes L, Jordan C, Esiashivilli N, Janns A, et al. Prolonged survival after treatment of diffuse intrinsic pontine glioma with radiation, temozolomide and bevacizumab: report of 2 cases. J Pediatr Hematol Oncol (2013) 35(1):e42-6. doi:10.1097/MPH.0b013e318279aed8

104. Rizzo D, Scalzone M, Ruggiero A, Maurizi P, Attina G, Mastrangelo S, et al. Temozolomide in the treatment of newly diagnosed diffuse brainstem glioma in children: a broken promise? J Chemother (2015) 27(2):106-10. doi:10.117 9/1973947814Y.0000000228

105. Warren KE. Diffuse intrinsic pontine glioma: poised for progress. Front Oncol (2012) 2:205. doi:10.3389/fonc.2012.00205

106. Veringa SJE, Biesmans D, van Vuurden DG, Jansen MHA, Wedekind LE, Horsman I, et al. In vitro drug response and efflux transporters associated with drug resistance in pediatric high grade glioma and diffuse intrinsic pontine glioma. PLoS One (2013) 8(4):e61512. doi:10.1371/journal.pone.0061512

107. Siegal T. Which drug or drug delivery system can change clinical practice for brian tumor therapy? Neuro Oncol (2013) 15(6):656-69. doi:10.1093/ neuonc/not016

108. Kemper EM, Boogerd W, Thuis I, Beijnen JH, van Tellingen O. Modulation of the blood-brain barrier in oncology: therapeutic opportunities for the treatment of brain tumours? Cancer Treat Rev (2004) 30:415-23. doi:10.1016/j. ctrv.2004.04.001

109. Siegal T, Rubinstein R, Bokstein F, Schwartz A, Lossos A, Shalom E, et al. In vivo assessment of the window of barrier opening after osmotic blood-brain barrier disruption in humans. J Neurosurg (2000) 92:599-605. doi:10.3171/jns.2000.92.4.0599

110. Prados MD, Schold SC, Fine HA, Jaeckle K, Hochberg F, Mechtler L, et al. A randomized, double-blind, placebo-controlled, phase 2 study of RMP-7 in combination with carboplatin administered intravenously for the treatment of recurrent malignant glioma. Neuro Oncol (2003) 5:96-103. doi:10.1093/ neuonc/5.2.96

111. Shukla S, Wu CP, Ambudkar SV. Development of inhibitors of ATP-binding cassette drug transporters-present status and challenges. Expert Opin Drug Metab Toxicol (2008) 4(2):205-23. doi:10.1517/17425255.4.2.205

112. Jones C, Perryman L, Hargrave D. Paediatric and adult malignant glioma: close relatives or distant cousins. Nat Rev Clin Oncol (2012) 9:400-13. doi:10.1038/nrclinonc.2012.87

113. Jansen MHA, van Vuurden DG, Vandertop WP, Kaspers GJL. Diffuse intrinsic pontine gliomas: a systematic update on clinical trials and biology. Cancer Treat Rev (2012) 38:27-35. doi:10.1016/j.ctrv.2011.06.007

114. Kebudi R, Cakir FB. Management of diffuse pontine gliomas in children: recent developments. Pediatr Drugs (2013) 15:351-62. doi:10.1007/ s40272-013-0033-5
115. Geoerger B, Hargrave D, Thomas F, Ndiaye A, Frappaz D, Andreiuolo F, et al. ITCC (innovative therapies for children with cancer) European consortium. Innovative therapies for children with cancer pediatric phase I study of erlotinib in brainstem glioma and relapsing/refractory brain tumors. Neuro Oncol (2011) 13(1):109-18. doi:10.1093/neuonc/noq141

116. Pollack IF, Stewart CF, Kocak M, Poussaint TY, Broniscer A, Banerjee A, et al. A phase II study of gefitinib and irradiation in children with newly diagnosed brainstem gliomas: a report from the pediatric brain tumor consortium. Neuro Oncol (2011) 13(3):290-7. doi:10.1093/neuonc/noq199

117. Geyer JR, Stewart CF, Kocak M, Broniscer A, Phillips P, Douglas JG, et al. A phase I and biology study of gefitinib and radiation in children with newly diagnosed brain stem gliomas or supratentorial malignant gliomas. Eur J Cancer (2010) 46(18):3287-93. doi:10.1016/j.ejca.2010.07.005

118. Massimino M, Bode U, Biassoni V, Fleischhack G. Nimotuzumab for pediatric intrinsic pontine gliomas. Expert Opin Biol Ther (2011) 11(2):247-56. do i: $10.1517 / 14712598.2011 .546341$

119. Bartels U, Wolff J, Gore L, Dunkel I, Gilheeney S, Allen J, et al. Phase 2 study of safety and efficacy of nimotuzumab in pediatric patients with progressive diffuse intrinsic pontine glioma. Neuro Oncol (2014) 16(11):1554-9. doi:10.1093/neuonc/nou091

120. Broniscer A, Baker JN, Tagen M, Onar-Thomas A, Gilbertson RJ, Davidoff $\mathrm{AM}$, et al. Phase I study of vandetanib during and after radiotherapy in children with diffuse intrinsic pontine glioma. J Clin Oncol (2010) 28(31):4762-8. doi:10.1200/JCO.2010.30.3545

121. Pollack IF, Jakacki RI, Blaney SM, Hancock ML, Kieran MW, Phillips P, et al. Phase I trial of imatinib in children with newly diagnosed brainstem and recurrent malignant gliomas: a pediatric brain tumor consortium report. Neuro Oncol (2007) 9:145-60. doi:10.1215/15228517-2006-031

122. Gururangan S, Chi SN, Poussaint TY, Onar-Thomas A, Gilbertson RJ, Vajapeyam S, et al. Lack of efficacy of bevacizumab plus irinotecan in children with recurrent malignant glioma and diffuse brainstem glioma: a pediatric brain tumor consortium study. J Clin Oncol (2010) 28:3069-75. doi:10.1200/JCO.2009.26.8789

123. Narayana A, Kunnakkat S, Chacko-Mathew J, Gardner S, Karajannis M, Raza $\mathrm{S}$, et al. Bevacizumab in recurrent high-grade pediatric gliomas. Neuro Oncol (2010) 12:985-90. doi:10.1093/neuonc/noq033

124. Salloum R, DeWire M, Lane A, Goldman S, Hummel T, Chow L, et al. Patterns of progression in pediatric patients with high-grade glioma or diffuse intrinsic pontine glioma treated with bevacizumab-based therapy at diagnosis. J Neurooncol (2015) 121:591-8. doi:10.1007/ s11060-014-1671-3

125. Geoerger B, Kieran MW, Grupp S, Perek D, Clancy J, Krygowski M, et al. Phase II trial of temsirolimus in children with high-grade glioma, neuroblastoma and rhabdomyosarcoma. Eur J Cancer (2012) 48:253-62. doi:10.1016/j. ejca.2011.09.021

126. Haas-Kogan DA, Banerjee A, Poussaint TY, Kocak M, Prados MD, Geyer JR, et al. Phase II trial of tipifarnib and radiation in children with newly diagnosed diffuse intrinsic pontine gliomas. Neuro Oncol (2011) 13(3):298-306. doi:10.1093/neuonc/noq202

127. MacDonald TJ, Stewart CF, Kocak M, Goldman S, Ellenbogen RG, Phillips $\mathrm{P}$, et al. Phase I clinical trial of cilengitide in children with refractory brain tumors: pediatric brain tumor consortium study PBTC-012. J Clin Oncol (2008) 26:919-24. doi:10.1200/JCO.2007.14.1812

128. Felix FHC, de Araujo OL, da Trindade KM, Trompieri NM, Fontenele BJ. Retrospective evaluation of the outcomes of children with diffuse intrinsic pontine glioma treated with radiochemotherapy and valproic acid in a single center. J Neurooncol (2014) 116:261-6. doi:10.1007/ s11060-013-1280-6

129. Grasso CS, Tang Y, Truffaux N, Berlow NE, Liu L, Debily MA, et al. Functionally defined therapeutic targets in diffuse intrinsic pontine glioma. Nat Med (2015) 21(7):827. doi:10.1038/nm0715-827a

130. Li G, Mitra SS, Monje M, Henrich KN, Bangs CD, Nitta RT, et al. Expression of epidermal growth factor variant III (EGFRvIII) in pediatric diffuse intrinsic pontine gliomas. J Neurooncol (2012) 108:395-402. doi:10.1007/ s11060-012-0842-3

131. Zhou Z, Luther N, Ibrahim GM, Hawkins C, Vibhakar R, Handler $\mathrm{MH}$, et al. B7-H3, a potential therapeutic target, is expressed in diffuse 
intrinsic pontine glioma. J Neurooncol (2013) 111:257-64. doi:10.1007/ s11060-012-1021-2

132. Pollack IJ, Jakacki RI, Butterfield LH, Hamilton RL, Panigrahy A, Potter DM, et al. Antigen-specific immune responses and clinical outcome after vaccination with glioma-associated antigen peptides and polyinosinic-polycytidylic acid stabilized by lysine and carboxymethylcellulose in children with newly diagnosed malignant brainstem and nonbrainstem gliomas. J Clin Oncol (2014) 32:2050-8. doi:10.1200/JCO.2013.54.0526

133. Warren KE, Poussaint TY, Vezina G, Hargrave D, Packer RJ, Stewart S, et al. Challenges with defining response to antitumor agents in pediatric neuro-oncology: a report from the response assessment in pediatric neuro-oncology (RAPNO) working group. Pediatr Blood Cancer (2013) 60:1397-401. doi: $10.1002 / p b c .24562$

134. Hipp S, Steffen-Smith E, Hammoud D, Shih J, Bent R, Warren K. Predicting outcome of children with diffuse intrinsic pontine gliomas using multi-parametric imaging. Neuro Oncol (2011) 13:904-9. doi:10.1093/ neuonc/nor076

135. Steffen-Smith EA, Venzon DJ, Bent RS, Hipp SJ, Warren KE. Single- and multivoxel proton spectroscopy in pediatric patients with diffuse intrinsic pontine glioma. Int J Radiat Oncol Biol Phys (2012) 84(3):774-9. doi:10.1016/j. ijrobp.2012.01.032

136. Jansen MH, van Zanten SEV, Aliaga ES, Heymans MW, Warmuth-Metz M, Hargrave D, et al. Survival prediction model of children with diffuse intrinsic pontine glioma based on clinical and radiological criteria. Neuro Oncol (2015) 17(1):160-6. doi:10.1093/neuonc/nou104
137. Hashizume R, Andor N, Ihara Y, Lerner R, Gan H, Chen X, et al Pharmacologic inhibition of histone demethylation as a therapy for pediatric brainstem glioma. Nat Med (2014) 20(12):1394-6. doi:10.1038/nm.3716

138. Funato K, Major T, Lewis PW, David Allis C, Tabar V. Use of human embryonic stem cells to model pediatric gliomas with $\mathrm{H} 3.3 \mathrm{~K} 27 \mathrm{M}$ histone mutation. Science (2014) 346(6216):1529-33. doi:10.1126/science.1253799

139. Aoki Y, Hashizume R, Ozawa T, Banerjee A, Prados M, David James C, et al. An experimental xenograft mouse model of diffuse pontine glioma designed for therapeutic testing. J Neurooncol (2012) 108:29-35. doi:10.1007/ s11060-011-0796-x

140. Saratsis AM, Kambhampati M, Snyder K, Yadavilli S, Devany JM, Harmon B, et al. Comparative multi-dimensional molecular analyses of pediatric diffuse intrinsic pontine glioma reveals distinct molecular subtypes. Acta Neuropathol (2014) 127:881-95. doi:10.1007/s00401-013-1218-2

Conflict of Interest Statement: The authors declare that the research was conducted in the absence of any commercial or financial relationships that could be construed as a potential conflict of interest.

Copyright (๑) 2015 Vanan and Eisenstat. This is an open-access article distributed under the terms of the Creative Commons Attribution License (CC BY). The use, distribution or reproduction in other forums is permitted, provided the original author(s) or licensor are credited and that the original publication in this journal is cited, in accordance with accepted academic practice. No use, distribution or reproduction is permitted which does not comply with these terms. 